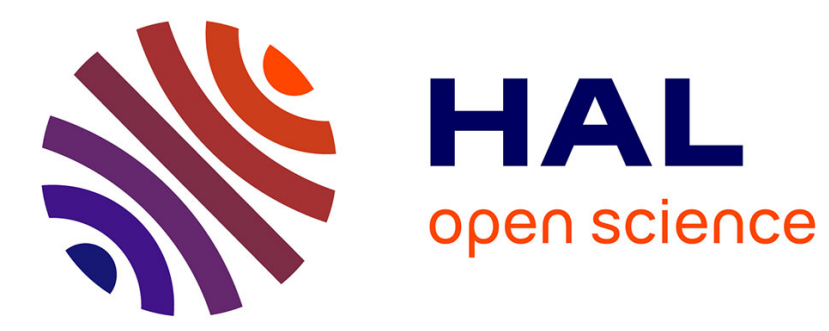

\title{
Passive scalar decay laws in isotropic turbulence: Prandtl number effects
}

\author{
Antoine Briard, Thomas Gomez, Pierre Sagaut, Souzan Memari
}

\section{To cite this version:}

Antoine Briard, Thomas Gomez, Pierre Sagaut, Souzan Memari. Passive scalar decay laws in isotropic turbulence: Prandtl number effects. Journal of Fluid Mechanics, 2015, 784, pp.274 - 303. 10.1017/jfm.2015.575 . hal-01429641

\section{HAL Id: hal-01429641 https://hal.sorbonne-universite.fr/hal-01429641}

Submitted on 9 Jan 2017

HAL is a multi-disciplinary open access archive for the deposit and dissemination of scientific research documents, whether they are published or not. The documents may come from teaching and research institutions in France or abroad, or from public or private research centers.
L'archive ouverte pluridisciplinaire HAL, est destinée au dépôt et à la diffusion de documents scientifiques de niveau recherche, publiés ou non, émanant des établissements d'enseignement et de recherche français ou étrangers, des laboratoires publics ou privés. 


\title{
Passive scalar decay laws in isotropic turbulence: Prandtl effects
}

\author{
A. Briard ${ }^{1}$, T. Gomez ${ }^{1} \dagger$, P. Sagaut ${ }^{2}$ \\ and S. Memari ${ }^{3}$ \\ ${ }^{1}$ Institut Jean Le Rond d'Alembert, CNRS UMR 7190, 4 Place Jussieu, F-75252 Paris \\ CEDEX 5, France \\ ${ }^{2}$ Aix-Marseille Université, CNRS, Centrale Marseille, M2P2 UMR 7340, 13451 Marseille, \\ France \\ ${ }^{3}$ Ecole Polytechnique, 91120 Palaiseau CEDEX, France
}

(Received 25 March 2015; revised 14 August 2015; accepted 25 September 2015 - Vol 784, $274-303)$

The passive scalar dynamics in a freely decaying turbulent flow is studied. The classical framework of homogeneous isotropic turbulence without forcing is considered. Both low and high Reynolds number regimes are investigated for very small and very large Prandtl numbers. The long time behaviours of integrated quantities such as the scalar variance or the scalar dissipation rate are analyzed by considering that the decay follows power laws. This study addresses three major topics. Firstly, the Comte-Bellot and Corrsin (CBC) dimensional analysis for the temporal decay exponents is extended to the case of a passive scalar when the permanence of large eddies is broken. Secondly, using numerical simulations based on eddy-damped quasi-normal markovian (EDQNM) model, the time evolution of integrated quantities is accurately determined for a wide range of Reynolds and Prandtl numbers. These simulations show that, whatever the Reynolds and the Prandtl numbers are, the decay follows an algebraic law with an exponent very close to the value predicted by the $\mathrm{CBC}$ theory. Finally, the initial position of the scalar integral scale $L_{T}$ has no influence on the asymptotic values of the decay exponents, and an analytical law predicting the relative positions of the kinetic and scalar spectra peaks is derived.

Key words: homogeneous turbulence, isotropic turbulence, scalar, decay Download link

\section{Introduction}

The evolution of a passive scalar such as temperature, injected in a freely decaying homogeneous isotropic turbulent flow, is still a challenging topic. Its dynamics is governed by the velocity field and various parameters such as the Reynolds and Prandtl numbers $R e$ and $\operatorname{Pr}=\nu / \kappa$, where $\nu$ is the kinematic viscosity and $\kappa$ the thermal diffusivity. Several publications such as Comte-Bellot \& Corrsin (1966); Kaneda (1986); Lesieur et al. (1987); Goto \& Kida (1999); Lesieur (2008) have focused on theoretical analysis and numerical simulations of the passive scalar evolution and it appears that there are still open questions, such as the behaviour of the scalar field with respect to the Prandtl number or the influence of the scale at which the scalar variance is injected.

$\dagger$ Email address for correspondence: thomas.gomez@upmc.fr 


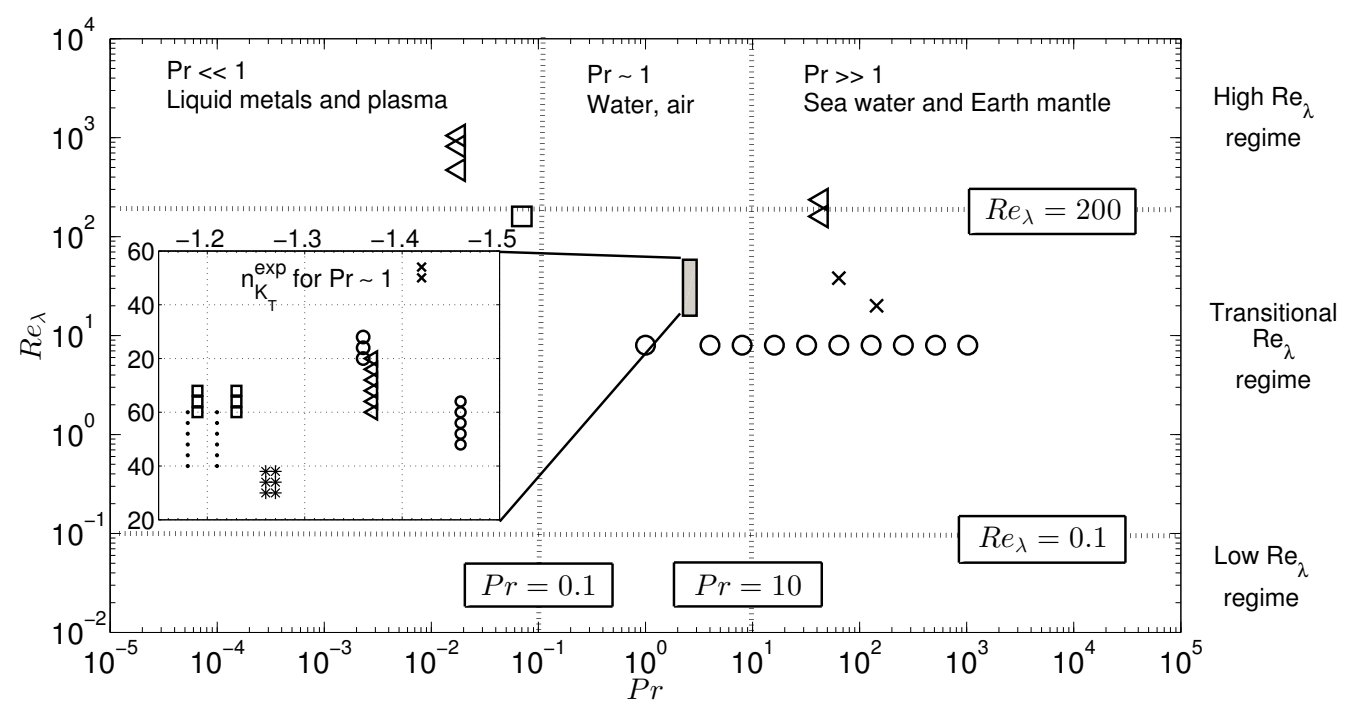

Figure 1. Schematic view of the $\left(R e_{\lambda}, \operatorname{Pr}\right)$ combinations used for the passive scalar decay analysis. On the main figure, triangles, squares, crosses and circles respectively refer to works of Rust and Sesonske (1966), Granatstein (1966), Yeung (2002) and Yeung (2004). On the zoom of the small grey region at $\operatorname{Pr} \sim 1$, experimental scalar decay exponents of Figure 10 are reported: dots, squares, stars, circles, triangles and crosses represent respectively works of Antonia (2013), Lee (2012), Antonia (2013) and Lee (2012), Zhou (2000), Antonia (2004) and Dainala (2000)

A crucial step toward the understanding of the passive scalar dynamics is the study of decay laws at very high or very small Prandtl number. Firstly, the decay permits to get ride of the forcing parameter, and secondly, the CBC dimensional analysis provides theoretical results concerning decay exponents of integrated quantities. Thus, for the passive scalar field, the comparison between the experimental and numerical decay exponents of integrated quantities (such as the scalar variance $K_{T}(t)$, the scalar integral scale $L_{T}(t)$ or the scalar dissipation rate $\left.\epsilon_{T}(t)\right)$ and the CBC theory could give interesting informations on what are the main phenomena which drive the scalar decay.

Several experimental works (Lin \& Lin (1973); Warhaft \& Lumley (1978); Sreenivasan et al. (1980); Danaila et al. (2000); Zhou et al. (2000, 2002); Lee \& Antonia (2012); Antonia et al. (2013)) and Direct Numerical Simulation (DNS) (Antonia \& Orlandi (2004)) have already focused on scalar decay exponents. However, these scalar decay exponents exhibit a very significant dispersion, as shown in Figure 1, whereas the experimental setups are designed to produce a very similar turbulent dynamics. There is up to $20 \%$ of discrepancy for similar Reynolds numbers and a fixed Prandtl number $(\operatorname{Pr} \sim 0.7)$. This scattering between the measured scalar decay exponents may be due to transitional effects coming from a Reynolds number not high enough, already studied in the kinetic case in Meldi \& Sagaut (2013), and also probably to the experimental turbulence production mechanisms which not permit to obtain a universal decay. A supplementary potential explanation for the scattering might be that the flow is not fully isotropic.

It is worth noting that the decay of the passive scalar has only been studied in experimental works and DNS in a small region of the $(R e, P r)$ map given in Figure 1. In this region (the small grey zone), the Prandtl number is close to one ( 0.7 for air which is the most used fluid) and the Taylor Reynolds number (Reynolds number based on Taylor 


\begin{tabular}{|c|c|c|c|c|}
\hline Reference & & $\operatorname{Re}$ & Predictions & \\
\hline Corrsin (1951) & & High & $K \propto t^{-10 / 7}$ & $K_{T} \propto t^{-6 / 7}$ \\
\hline Corrsin (1951) & & Low & $K \propto t^{-5 / 2}$ & $x t^{-3 / 2}$ \\
\hline Nelkin \& Kerr $(19$ & & High & $K \propto t^{-6 / 5}$ & $K_{T} \propto t^{-6 / 5}$ \\
\hline Ristorcelli \& Livescu & $2004)$ & High & $K \propto t^{-1}$ & $K_{T} \propto t^{-1}$ \\
\hline
\end{tabular}

TABle 1. Decay exponents for the kinetic energy $K=<u_{i} u_{i}>/ 2$ and scalar variance $K_{T}=<T^{2}>/ 2$ at $\operatorname{Pr} \sim 1$

scale) is such that $R e_{\lambda} \leqslant 70$. The values of the decay exponents obtained in these works will be reported in Figure 10 along with our numerical simulations. Other experiments and DNS performed for Prandtl numbers different from one, spanning from $10^{-2}$ to $10^{2}$ in Figure 1 (Rust \& Sesonske (1966); Granatstein \& Buchsbaum (1966); Yeung et al. (2004)) only focus on the shape of the scalar spectrum $E_{T}(k, t)$ and not on the decay of the passive scalar itself. Therefore, they cannot be exploited for comparison purpose in the present study.

Various theoretical decay exponents for the kinetic energy and scalar variance (Nelkin \& Kerr (1981); Ristorcelli \& Livescu (2004); Ristorcelli (2006)) have been gathered in Table 1. The analytical study of Nelkin is performed in the particular case of Saffman turbulence (infrared exponents of the kinetic and scalar spectra are set equal to 2): from a Richardson law, the same result as the CBC theory is derived. As for Ristorcelli, a decay law in $K_{(T)} \propto t^{-1}$ is derived using assumptions on statistical quantities of the flow (kinetic and scalar skewness and palinstrophy). However this behavior is observed in the EDQNM simulations only for the initial infrared exponents $s=1$.

When the Prandtl number departs from unity, as described in Tennekes \& Lumley (1972), dimensional analysis shows that at large or small Prandtl number, the shape of the scalar spectrum $E_{T}(k, t)$ is significantly modified at scales smaller than the integral scale. Nevertheless, as illustrated in Figure 1, the regions where $\operatorname{Pr} \ll 1$ and $\operatorname{Pr} \gg 1$ have not been much explored for the passive scalar decay issue. Hence, an interesting question could be: does the Prandtl number, in addition to the Reynolds number and initial conditions, modify the decay laws of scalar integrated quantities $K_{T}(t), L_{T}(t)$ and $\epsilon_{T}(t)$ ? This paper aims at answering this question of interest for the understanding of the passive scalar dynamics, and at providing an explanation to the scattering of experimental scalar decay exponents.

To this end, this paper focuses on two main approaches. The first one is based on the dimensional analysis of Comte-Bellot and Corrsin that is extended to a more general case for the passive scalar, following the work of Meldi \& Sagaut (2012) in the kinetic case. The second approach relies on the EDQNM analysis (developed by Orszag (1970); Leith (1971); Orszag (1977) and extended to the scalar case in Herring et al. (1982)) to perform numerical simulations of the turbulent mixing. The main advantage of this method is its accuracy and low cost in investigating the turbulent dynamics for a broad range of Reynolds and Prandtl numbers. EDQNM simulations have already been used to study kinetic decay exponents (Lesieur \& Ossia (2000); Meldi \& Sagaut (2012, 2013)) and passive scalar dynamics (Lesieur et al. (1987)). Moreover, this method also allows to recover theoretical results regarding the scalar spectrum shape when $\operatorname{Pr} \leqslant 1$ and $\operatorname{Pr} \geqslant 1$ that have been obtained in experiments and DNS (Chasnov et al. (1988); Lesieur \& Rogallo (1989); Antonia \& Orlandi (2004); Yeung et al. (2004); Briard \& Gomez (2015)).

Hereafter, new theoretical and numerical arguments are proposed to understand how 


$\begin{array}{lcccccc}\text { Reynolds } & \text { Cases } & n_{K}, n_{K} & n_{\varepsilon}, n_{\varepsilon_{T}} & n_{L}, n_{L_{T}} & n_{R e_{L}} & n_{R e_{\lambda}} \\ \text { High } & \text { Kinetic } & -2 \frac{s-p+1}{s-p+3} & -3 \frac{s-p+5 / 3}{s-p+3} & \frac{2}{s-p+3} & -\frac{s-p-1}{s-p+3} & -\frac{1}{2} \frac{s-p-1}{s-p+3} \\ & \text { Scalar } & -2 \frac{s^{\prime}-p^{\prime}+1}{s-p+3} & -\frac{s-p+5+2 s^{\prime}-2 p^{\prime}}{s-p+3} & \frac{2}{s-p+3} & & \\ \text { Low } & \text { Kinetic } & -\frac{s+1}{2} & -\frac{s+3}{2} & \frac{1}{2} & -\frac{s-1}{4} & -\frac{s-1}{8} \\ & \text { Scalar } & -\frac{s^{\prime}+1}{2} & -\frac{s^{\prime}+3}{2} & \frac{1}{2} & \end{array}$

TABLE 2. Kinetic and scalar exponents for classical and extended CBC analysis. $K$ and $K_{T}$ are the kinetic energy and scalar variance, $\epsilon$ and $\epsilon_{T}$ the kinetic and scalar dissipation rates, $L$ and $L_{T}$ the kinetic and scalar integral scales, $R e_{L}$ the integral Reynolds number and $R e_{\lambda} \sim \sqrt{R e_{L}}$ the Reynolds number based on Taylor microscale. $s$ and $s^{\prime}$ are the kinetic and scalar infrared slopes, and $p$ and $p^{\prime}$ backscatter parameters for the kinetic and scalar spectrum respectively. All the expressions with $s^{\prime}$ and $p^{\prime}$ are new results and will be obtained in the following sections.

the decay laws of the passive scalar field behave with a Prandtl number different from one. After describing the EDQNM modelling in $\$ 2$, the CBC theory is extended to a more general framework in $\$ 3$. Results obtained on the base of the CBC dimensional analysis are summarized in Table 2, where all the expressions using the parameters $s^{\prime}$ and $p^{\prime}$ correspond to the original scalar extension presented in this paper. Then, in 4 and $\$ 5$. decay exponents of scalar quantities are compared to the theoretical predictions of the CBC dimensional analysis, for both high and low Reynolds numbers. The transition between these two regimes is investigated as well for high and low Prandtl numbers. Finally, in $\$ 6$, the influence of the relative initial position of the kinetic and scalar integral scales on the dynamics is addressed. A law that can predict the relative position of the kinetic and scalar spectra peaks is derived.

\section{EDQNM modelling}

The isotropic homogeneous freely decaying turbulence is analyzed in the present paper using the EDQNM model first introduced by Orszag (1970, 1977) and Leith (1971) for hydrodynamic turbulence. In this section, a brief description of the EDQNM model is given along with the associated numerical procedure. This model is used to solve the equations of dynamics in order to obtain the time evolution of the kinetic and scalar spectra. More details about the EDQNM theory are given in the Appendix $\mathrm{A}$ and the reader is referred to Lesieur (2008) and Sagaut \& Cambon (2008) for more details.

The EDQNM model is a quasi-normal closure. This approximation supposes that the fourth-order moments of the velocity field appearing in the evolution equation of the third-order correlations are related to the second-order moments as if the field was Gaussian. The realizability of the model is then enforced by adding an eddy-damping term

$$
\eta^{E D}(k, t)=a_{1} \sqrt{\int_{0}^{k} p^{2} E(p, t) d p}
$$

that reflects departure from Gaussianity, where $a_{1}=0.36$ is set to recover the Kolmogorov constant $K_{0} \simeq 1.40$. The Markovianization leads to define the relaxation time of the third-order moments in the EDQNM model as

$\theta_{k p q}=\frac{1-e^{-\mu_{k p q} t}}{\mu_{k p q}} \quad$ where $\quad \mu_{k p q}=\nu\left(k^{2}+p^{2}+q^{2}\right)+\eta^{E D}(k, t)+\eta^{E D}(p, t)+\eta^{E D}(q, t)$. 


$\begin{array}{lcccc}\text { Simulation } & R e_{\lambda}(t=0) & \operatorname{Pr} & C F L & k_{\min } \\ \text { Series A } & 2340 & 1 & 0.3 & 10^{-5} \\ \text { Series C } & 2340 & 10-10^{5} & 0.3 & 10^{-5} \\ \text { Series D } & 23330 & 10^{-5}-0.1 & 0.6 & 10^{-5} \\ \text { Series B } & 240 & 1 & 0.3 & 10^{-10} \\ \text { Series E } & 240 & 10-10^{5} & 0.3 & 10^{-10} \\ \text { Series F } & 1620 & 10^{-5}-0.1 & 0.6 & 10^{-10}\end{array}$

TABLE 3. Physical and numerical parameters used for the simulations

Additional details concerning this closure are given in the Appendix A, especially regarding the scalar field.

The time evolution of the kinetic and scalar spectra $E(k, t)$ and $E_{T}(k, t)$ is obtained by solving two coupled integro-differential equations using a third order Runge Kutta scheme with implicit treatment of viscous terms. The wavenumber space is discretized using a logarithmic mesh $k_{i+1}=r k_{i}$ for $i=1, \ldots, n$, where $n$ is the total number of modes and $r=10^{1 / f}, f=17$ being the number of points per decade. This mesh spans from $k_{\text {min }}$ to $k_{\max }=10 k_{\eta_{T}}$ with $k_{\eta_{T}}=k_{B}$ if $\operatorname{Pr} \geqslant 1$ or $k_{\eta_{T}}=k_{C O}$ if $\operatorname{Pr} \leqslant 1$. Both wavenumbers $k_{B}$ and $k_{C O}$ will be defined hereafter. The time step $\Delta t$ is monitored by setting a constant $C F L$ number and is obtained by considering the characteristic time scales of scalar and kinetic dynamics leading to $\Delta t=\frac{C F L \cdot R e}{20} \min \left\{\frac{1}{k_{\eta}^{2}}, \frac{P r}{k_{\eta_{T}}^{2}}\right\}$. Hence the time evolution of $\Delta t$ is consistent with the physical evolution of the turbulence even for very large time when the Taylor Reynolds number becomes small, i.e. $R e_{\lambda} \sim 10^{-2}$. The values of the physical and numerical parameters are given for simulations at large and small Reynolds number in Table 3 .

The initial kinetic and scalar spectra $E$ and $E_{T}$ are isotropic and the shape was proposed by Pope (2000); Meyers \& Meneveau (2008) as

$$
E(k, t=0)=K_{0} k^{-5 / 3} \epsilon^{2 / 3} f_{L}(k L) f_{\eta}(k \eta)
$$

where $f_{L}$ and $f_{\eta}$ are shape functions for large and small scales respectively

$$
f_{L}(x)=\left(\frac{x}{\left(x^{1.5}+1.5-s / 4\right)^{2 / 3}}\right)^{\frac{5}{3}+s}, f_{\eta}(x)=\exp \left(-5.3\left(\left(x^{4}+0.4^{4}\right)^{\frac{1}{4}}-0.4\right)\right) .
$$

Different initial conditions were tested and there were no difference observed in the final decay regime.

\section{Comte-Bellot Corrsin dimensional analysis: scalar extension}

In this section, the case $\operatorname{Pr}=1$ is considered for which diffusive and dissipative effects are of the same order. The influence of the Prandtl number on the decay will be discussed below in $\$ 4$ and \$5. Here, the CBC theory ( $c f$. Comte-Bellot \& Corrsin (1966)) based on the dimensional analysis, and recently extended by Meldi \& Sagaut (2012) in the purely kinetic case when the permanence of large eddies is broken, is briefly recalled. In this paper, a new correction is proposed when the infrared scalar spectrum power law exponent is $s^{\prime}=4\left(E_{T}\left(k<k_{T}, t\right) \sim k^{s^{\prime}}\right)$. Firstly, the basic equations of the homogeneous isotropic turbulence useful for the dimensional analysis are recalled. 


\subsection{Equations of the homogeneous isotropic turbulence}

The turbulent kinetic energy of the fluctuating velocity $u_{i}$ is defined as $K=\frac{1}{2}<u_{i} u_{i}>$ and the variance of temperature fluctuations $T$ as $K_{T}=\frac{1}{2}\left\langle T^{2}\right\rangle$, where $\left\langle\cdot{ }^{2}\right\rangle$ denotes an ensemble average. Introducing the mean kinetic and scalar dissipation, respectively noted $\epsilon(t)$ and $\epsilon_{T}(t)$, one can write the governing equations for these global quantities $K(t)$ and $K_{T}(t)$ in a freely-evolving flow as

$$
\frac{d K}{d t}=-\epsilon \quad \text { and } \quad \frac{d K_{T}}{d t}=-\epsilon_{T} .
$$

In turbulence, the highly diffusive flow contains a wide range of active and dynamic scales. Indeed the velocity field fluctuations induce a rapid mixing of all injected passive scalar. In order to characterize the dynamics of turbulent flows, several characteristic scales associated with physical mechanisms are identified. In the freely decaying isotropic case, the integral scale $L(t)$ carries most of the kinetic energy of the flow and can be interpreted as the characteristic size of large eddies. At Kolmogorov scale $\eta=\left(\nu^{3} / \epsilon\right)^{1 / 4}$, viscous effects become dominant compared to the inertial ones, and thus $E(k, t)$ rapidly vanishes. The equivalent scales for the scalar field are the scalar integral scale $L_{T}(t)$, the Corrsin-Obukhov scale $\eta_{C O}=\left(\kappa^{3} / \epsilon\right)^{1 / 4}=\mathrm{Pr}^{-3 / 4} \eta$, beyond which diffusion of the scalar become dominant, and the Batchelor scale $\eta_{B}=\left(\nu \kappa^{2} / \epsilon\right)^{1 / 4}=\operatorname{Pr}^{-1 / 2} \eta$, beyond which the scalar fluctuations are no longer agitated by small scale convection. In spectral space, both dissipative and diffusive scales are represented respectively by the wavenumbers $k_{\eta}=1 / \eta, k_{C O}=1 / \eta_{C O}$ and $k_{B}=1 / \eta_{B}$. As the scalar field dynamics is only driven by the velocity field, one can naturally use the Prandtl number $\operatorname{Pr}=\nu / \kappa$ to distinguish the three main physical regimes, i.e. $\operatorname{Pr} \ll 1, \operatorname{Pr}=\mathcal{O}(1)$ and $\operatorname{Pr} \gg 1$. The behaviour of the scalar spectrum has been derived using phenomenological arguments in Tennekes \& Lumley (1972).

Using these scales, the shape of the scalar variance spectrum $E_{T}$ and fluctuating kinetic energy spectrum $E$ can be determined by dimensional analysis. Kinetic and scalar energies and dissipation rates are linked to the spectra through

$$
\begin{array}{cc}
K(t)=\int_{0}^{\infty} E(k, t) d k, & K_{T}(t)=\int_{0}^{\infty} E_{T}(k, t) d k \\
\epsilon(t)=2 \nu \int_{0}^{\infty} k^{2} E(k, t) d k, & \epsilon_{T}(t)=2 \kappa \int_{0}^{\infty} k^{2} E_{T}(k, t) d k .
\end{array}
$$

Different power-law ranges for the small scales of $E_{T}$ have been observed in numerical simulation (Chasnov et al. (1988)) and in experimental works as well (Sreenivasan (1996); Grant et al. (1968)). These power-law ranges are consistent with the abridged LHDIA (Kraichnan (1968)), DIA (Goto \& Kida (1999)) or LRA Kaneda 1986) theories. Moreover, all these ranges, present in the stationary turbulence case, can also be observed in the freely decaying turbulence framework.

\subsection{Decay law for the kinetic case in high Reynolds regime}

In the $\mathrm{CBC}$ theory the shape of the kinetic spectrum is considered as

$$
E(k)= \begin{cases}A(t) k^{s} & \text { for } k \leqslant k_{L} \text { and } 1 \leqslant s \leqslant 4 \\ K_{0} \epsilon^{2 / 3} k^{-5 / 3} & \text { for } k_{L} \leqslant k \leqslant k_{\eta}\end{cases}
$$

according to the Kolmogorov pioneering work for the inertial range. $k_{L} \simeq 1 / L$ is the peak of the kinetic energy spectrum and $K_{0} \simeq 1.40$ is the Kolmogorov constant. The $k^{-5 / 3}$ zone is the inertial range which goes from the wavenumber associated to the integral 
scale $k_{L}=1 / L$ to the Kolmogorov wavenumber $k_{\eta}$. The shape at large scales $\left(k<k_{L}\right)$ is referred to as the infrared range. The scaling of the kinetic energy spectrum $E \sim k^{s}$ is given by theoretical arguments in Batchelor \& Proudman (1956). Initial conditions with $s>4$ (such as a peak of energy around the integral scale) result in a large scale spectrum in $k^{4}$ (Lesieur \& Schertzer 1978; Lesieur \& Ossia 2000). Whereas for a forcedturbulence, the infrared range behaves in $k^{2}$ (Saffman (1967); Lumley (1970)). More recently, it has been shown that only the slope near the peak of energy $k_{L}$ (and not for $k \rightarrow 0$ ) is important and leads the decay (Meldi \& Sagaut 2012; Mons et al. 2014). Finally, according to Llor \& Soulard (2013), it seems that any positive real values such that $s \leqslant 4$ could be physically acceptable.

The original method of $\mathrm{CBC}$ is based on the concept of invariance of very large scale eddies corresponding to $k<k_{L}$ which is notably valid for infrared slopes $s=1,2$ and 3 . In fact in the kinetic case, the value of the infrared spectral slope $s$ is time-independent and remains constant for $s=1,2$ (Saffman), 3 and 4 (Batchelor). As for the coefficient $A(t)$ of the infrared spectrum, it remains constant for values of $s \in\{1,2,3\}$, and evolves in time as $A(t) \propto L(t)^{p}$ in the case $s=4$.

By dimensional analysis in the case of high Reynolds regime (when there is an inertial zone), one obtains

$$
n_{K}(s, p)=-2 \frac{(s+1-p)}{(s+3-p)} \quad \text { where } \quad K(t) \propto t^{n_{K}}
$$

in which $p=0$ if $s \leqslant 3$ and $p \sim 0.55$ if $s=4$ as computed in Lesieur et al. (1987); Eyink \& Thomson (2000); Meldi \& Sagaut (2012). Other kinetic exponents such as $n_{\epsilon}$ and $n_{L}$ can also be determined using (3.3) and are given in Table 2.

\subsection{Scalar extended CBC analysis in high Reynolds and Péclet regimes}

The relevant parameter to study the dynamics of the passive scalar is not only the Reynolds number, but also the Péclet number $P e=R e P r$. In this section, the emphasis is put on the case $s^{\prime}=4$. Numerical simulations show that the scalar variance also decreases with time following a power law

$$
K_{T}(t)=\frac{1}{2}<T^{2}>\propto t^{n_{K_{T}}}
$$

After a transient relaxation phase, the kinetic and scalar characteristic length scales exhibit the same behaviour and their decay exponents $n_{L}$ and $n_{L_{T}}$ converge toward the same value as shown in Figure 2 Consequently, we consider that $n_{L}=n_{L_{T}}$, and choose as an initial condition $L(t=0)=L_{T}(t=0)=1$ to analyze the decay of the scalar field. It will be shown in $\$ 6$ that the relative initial position of $L$ and $L_{T}$ has no consequence for the final decay power-laws, and this will justify a posteriori the present choice.

At very large scales, the passive scalar field is fully controlled by the velocity one. The scalar spectrum $E_{T}(k, t)$ admits an asymptotic behaviour similar to $E(k, t)$

$$
E_{T}\left(k<k_{T}, t\right) \propto k^{s^{\prime}} \quad \text { where } \quad s^{\prime} \in[1,4],
$$

where $k_{T} \simeq 1 / L_{T}$ is the peak of the scalar spectrum. The scalar variance spectrum depends on various physical quantities: the Prandtl number $\mathrm{Pr}$, the scalar dissipation $\epsilon_{T}$ and the kinetic energy dissipation $\epsilon$. Therefore, using dimensional analysis the scalar spectrum can be written as

$$
E_{T}(k, t)= \begin{cases}A_{T}(t) k^{s^{\prime}} & \text { if } \quad k \leqslant k_{T} \\ K_{C O} \epsilon_{T} \varepsilon^{-1 / 3} k^{-5 / 3} & \text { if } \quad k_{T} \leqslant k \leqslant k_{\eta}\end{cases}
$$



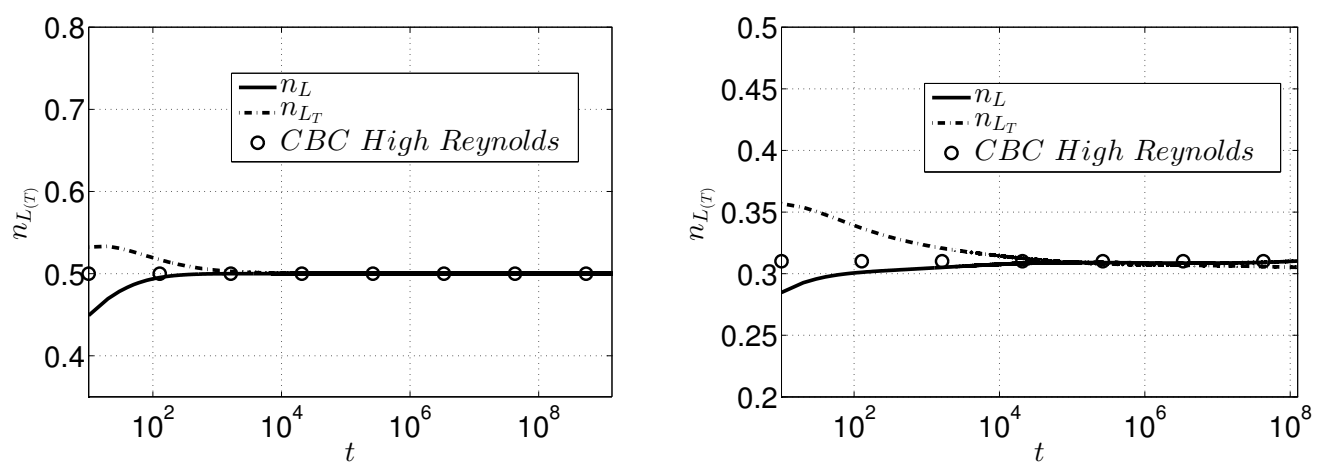

Figure 2. Convergence of the two scalar and kinetic characteristic lengths' exponents $n_{L}$ and $n_{L_{T}}$ at $\operatorname{Pr}=1$ for two cases. At left, $s=s^{\prime}=1$. At right, $s=s^{\prime}=4$

where $K_{C O} \simeq 1.66$ is the Corrsin-Obukhov constant. In the case of a Prandtl number of order one, the scalar dynamics is exclusively dictated by the velocity field at all scales of the spectrum. This is why it exhibits a $k^{-5 / 3}$ inertial range similar to the kinetic spectrum, called the inertial-convective range, when $k_{T} \leqslant k \leqslant k_{\eta}$. As mentioned in $\$ 1$, the shape of both scalar and kinetic spectra exhibits the same behaviours in terms of power law exponents. Therefore the coefficient $A_{T}(t)$ varies as $L_{T}^{p^{\prime}}$ where $p^{\prime}$ only depends on $s$ and $s^{\prime}$ as revealed in Table 4

By writing the continuity of scalar spectrum at wavenumber $1 / L_{T}(t)$ and considering that the passive scalar does not affect the kinetic part of the flow, one can use the expressions given in Table $2 n_{L}=\frac{2}{s-p+3}$ and $n_{\epsilon}=-3 \frac{s-p+5 / 3}{s-p+3}$ and conclude that

$$
\epsilon_{T} \propto t^{n_{\epsilon_{T}}} \quad \text { with } \quad n_{\epsilon_{T}}\left(s, s^{\prime}, p, p^{\prime}\right)=-\frac{s-p+5+2\left(s^{\prime}-p^{\prime}\right)}{s-p+3} .
$$

Moreover, as the scalar dissipation is the time derivative of scalar variance, the exponent of the scalar variance is

$$
n_{K_{T}}\left(s, s^{\prime}, p, p^{\prime}\right)=-2 \frac{s^{\prime}-p^{\prime}+1}{s-p+3} .
$$

Details of the derivation of scalar exponents are very similar to those for the kinetic exponents. All corrected exponents for both kinetic and scalar cases are gathered in Table 2. From the theoretical decay exponents $n_{K}$ and $n_{K_{T}}$, it seems that a decay in $K_{(T)} \sim t^{-1}$ (Ristorcelli 2006) occurs only for $s=s^{\prime}=1$ (Meldi \& Sagaut 2013) and corresponds to a constant Reynolds number.

\subsection{Evaluation of $p^{\prime}$ with EDQNM}

When setting the infrared scalar spectrum slope to $s^{\prime}=4$, a significant departure from the exponents obtained with the classical CBC theory and EDQNM simulations is observed. The exponent $n_{K_{T}}$ obtained by the classical CBC theory and EDQNM simulations are plotted in Figure 3 for $s \in\{1,2,3,4\}$. The observed discrepancy is essentially due to the fact that the $\mathrm{CBC}$ analysis of the passive scalar case assumes that the coefficient $A_{T}(t)$ in (3.6) is constant. But the time evolution of the scalar spectrum shows that this coefficient evolves. Therefore, the parameter $p^{\prime}$ cannot be considered as zero anymore for $s^{\prime}=4$. It strongly depends on the kinetic infrared slope $s$ and slightly on $\operatorname{Pr}$ (see Table 44. The extended CBC analysis, also plotted in Figure 3 , assesses the extension proposed in the previous part. 


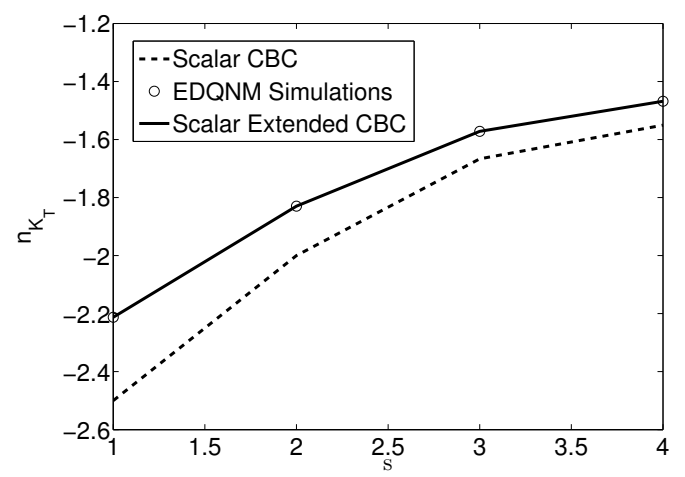

FIGURE 3. $n_{K_{T}}$ as function of $s$ obtained by EDQNM and CBC analysis when $s^{\prime}=4$ and $\operatorname{Pr}=1$

$\begin{array}{ccccccc}s & \operatorname{Pr}=10^{-4} & \operatorname{Pr}=10^{-2} & \operatorname{Pr}=1 & \operatorname{Pr}=10 & \operatorname{Pr}=10^{2} & \operatorname{Pr}=10^{5} \\ 1 & & 0.5746 & 0.5745 & 0.5745 & & \\ 2 & & 0.4285 & 0.4254 & 0.4269 & & \\ 3 & & 0.2961 & 0.2842 & 0.2904 & & \\ 4 & 0.3275 & 0.2814 & 0.2652 & 0.2754 & 0.3125 & 0.3495\end{array}$

TABLE 4. Some values of $p^{\prime}$ for various $\operatorname{Pr}$ and $s$ with $s^{\prime}=4$.

Considering the case $s^{\prime}=4$ and variable infrared kinetic energy spectrum slope $s$, the values of $p^{\prime}$ are evaluated by averaging the coefficient obtained with different exponents as $n_{K_{T}}$ and $\epsilon_{T}$. The estimated error on the evaluation of this parameter is around $0.25 \%$. Hence, the values of $p^{\prime}$ are computed for various Prandtl numbers and gathered in the Table $4 p^{\prime}$ decreases when $s$ increases at a fixed $\operatorname{Pr}$, and $p^{\prime}$ increases when $\operatorname{Pr}$ departs from unity.

\subsection{Decay laws for kinetic and scalar cases in low Reynolds regime}

Here, the emphasis is put on the dynamics of the scalar variance considering small Reynolds numbers at $\operatorname{Pr}=1$. In the case of small Reynolds numbers, the inertial effects are rather small, and so inertial zone contributions to the kinetic and scalar spectra become negligible. Thus, the kinetic energy behaviour is assumed to be mainly determined by the contribution of the spectrum at large scales

$$
K(t)=\int_{0}^{\infty} E(k, t) d k \approx \int_{0}^{k_{L}} C k^{s} d k .
$$

By dimensional analysis $L(t)$ varies as $\sqrt{\nu t}$ and so $n_{L}=1 / 2$. This leads to

$$
K(t) \approx \int_{0}^{k_{L}} C k^{s} d k=\frac{C}{s+1} \nu^{-(s+1) / 2} t^{-(s+1) / 2} .
$$

Hence, from the kinetic energy $K$, the dissipation rate and other important decay exponents can be deduced, all gathered in Table 2. One obtains the exponent of the scalar variance using the relation $L_{T}(t) \propto \sqrt{\kappa t}$. Then, the integration of the scalar spectrum 
yields

and

$$
K_{T}(t)=\int_{0}^{\infty} E_{T}(k, t) d k \approx \int_{0}^{k_{T}} C_{T} k^{s^{\prime}} d k
$$

$$
K_{T}(t) \approx \int_{0}^{k_{T}} C_{T} k^{s^{\prime}} d k=\frac{C_{T}}{s^{\prime}+1} \kappa^{-\left(s^{\prime}+1\right) / 2} t^{-\left(s^{\prime}+1\right) / 2} .
$$

The other scalar decay exponents derived from $n_{K_{T}}$ are also presented in Table 2 . For instance, $n_{\epsilon_{T}}$ is simply computed using $d / d t\left(K_{T}\right)=\epsilon_{T}$. In what follows, all exponents are calculated using the EDQNM simulations for both $s$ and $s^{\prime}$ in the set $\{1,2,3,4\}$. For the kinetic case, there is an exact superposition of kinetic exponents with those of Lesieur \& Ossia (2000) and Meldi \& Sagaut (2012). For the scalar case, the EDQNM results for $s^{\prime} \leqslant 4$ are also significantly close (within $0.25 \%$ ) to the values of the CBC analysis.

\section{Scalar decay at high Reynolds numbers - Re $\gg 1$}

The emphasis is now put on the case where the initial Reynolds number is sufficiently high to allow the kinetic and scalar spectra $E$ and $E_{T}$ to decrease according to the extended $\mathrm{CBC}$ exponents given on the two first lines of Table 2. It will be shown in $\$ 6$ that the initial position of $L_{T}$ is not a governing parameter. Therefore, the initial condition $L(t=0)=L_{T}(t=0)$ to analyze the effect of $\operatorname{Pr}$ on the decay exponents is relevant. This section is divided into two parts. Firstly, the case of a very conductive passive scalar field $\operatorname{Pr} \leqslant 1$ is investigated, and then the opposite case where convection dominates $\operatorname{Pr} \geqslant 1$.

\subsection{Scalar field with high diffusivity: $\operatorname{Pr} \leqslant 1$}

\subsubsection{General study - Spectrum and decay}

In this section, scalar decay exponents provided by the extended CBC theory are found to be still valid in the case where $\operatorname{Pr} \ll 1($ i.e. $\nu \ll \kappa)$. Indeed, for $\operatorname{Pr} \sim 1, E_{T}$ behaves as $E$ and all decay exponents follow the CBC extended theory whatever $s$ and $s^{\prime}$ are. In the case of a passive scalar with a strong diffusivity $\kappa$ and in the asymptotic case of high Reynolds numbers, the shape of $E_{T}$ is well-attested. Due to the high thermal diffusion, the temperature undergoes a rapid decrease. In this case, a more complex analysis, described by Batchelor et al. (1958), is needed to obtain the scalar spectrum shape

$$
E_{T}(k, t)=\frac{K_{0}}{3} \epsilon_{T} \varepsilon^{2 / 3} \kappa^{-3} k^{-17 / 3} \quad \text { for } \quad k_{C O}<k<k_{\eta} .
$$

The range $k \in\left[k_{C O} ; k_{\eta}\right]$ is the inertial-diffusive range. The $k^{-17 / 3}$ spectrum was assessed by DNS in Chasnov et al. (1988), already observed with EDQNM in Marinis et al. (2013), and recovered here in Figure 4. There, the inertial-diffusive subrange grows in size when $\operatorname{Pr}$ decreases. One can observe that for $\operatorname{Pr}=10^{-5}$ there is only one decade left for the $k^{-5 / 3}$ range. The theoretical CBC exponents being based on the existence of an inertialconvective range, cases where $\operatorname{Pr}<10^{-5}$ will not be investigated: indeed, for decreasing $\operatorname{Pr}$ at a fixed Reynolds number, the inertial-convective range shrinks even though the kinetic spectrum still displays an inertial range. Near the Kolmogorov wavenumber $k_{\eta}$, transfers slow down and thus scalar energy accumulates. This phenomenon is studied in detail in Briard \& Gomez (2015).

In this part, the initial Reynolds number is $\operatorname{Re}_{\lambda}(0)=23330$, high enough to make sure that $P e=\operatorname{Re} \operatorname{Pr} \gg 1$. As $E_{T}(k, t)$ is located under $E(k, t)$, local energy transfers 


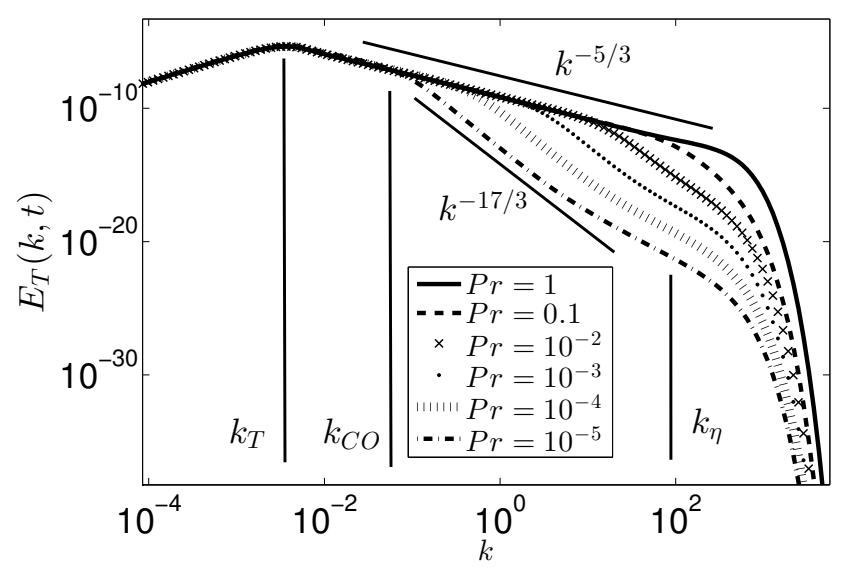

FIgURE 4. Scalar spectrum $E_{T}$ for $s=s^{\prime}=2$, with $k_{L}$ and $k_{\eta}$ for $\operatorname{Pr}=10^{-n}, n \in \llbracket 0 ; 5 \rrbracket$, and $k_{C O}$ for $\operatorname{Pr}=10^{-5}$. The Reynolds number is $\operatorname{Re} e_{\lambda}=5.10^{3}$.

dominate. Therefore, non-local transfers $T_{T}^{N L}$ defined in Appendix A are negligible. Decay exponents of $\epsilon_{T}, L_{T}$ and $K_{T}$ are investigated in Figure 5 for $s=s^{\prime}=2$ and $s=s^{\prime}=4$. The scalar decay exponents clearly follow the extended CBC theory. The result is the same for any $\operatorname{Pr} \leqslant 1$ as soon as the Péclet number is large enough.

\subsubsection{Remarks on large scales - Backscatter and non-local transfers}

The influence of non-local transfers and Prandtl number on the infrared subrange is briefly discussed. In Figure 6, with and without non-local transfers $T_{T}^{N L}$, the scalar spectrum $E_{T}$ does not change, which is consistent with the previous remark: local transfers dominate. In the next section where $\operatorname{Pr} \gg 1$, non-local transfers strongly affect small scales.

Moreover, EDQNM simulations show that the Prandtl number does not influence the backscatter effect $\left(s=4\right.$ or $\left.s^{\prime}=4\right)$ for $\operatorname{Pr} \in\left[10^{-5} ; 1\right]$. This means that $\operatorname{Pr}$ affects only small scales, and that the infrared slopes $s$ and $s^{\prime}$ are the sole relevant parameters at large scales.

\subsection{Scalar field with low diffusivity: $\operatorname{Pr} \geqslant 1$}

\subsubsection{General study - Spectrum and decay}

In this section, the case $\operatorname{Pr} \gg 1$ is finally addressed, and scalar decay exponents provided by the extended CBC theory are shown to be still valid.

In the asymptotic case of high Reynolds numbers and low diffusivity $\kappa$, the scalar spectrum $E_{T}$ behaves as $k^{-1}$ at very small scales. This slope is achieved by dimensional analysis, considering that the velocity fluctuations are strongly damped by viscous effects while diffusion does not affect the temperature fluctuations. The dynamics of temperature fluctuations is then controlled by shear and friction with the characteristic time scale $\tau_{B}=(\nu / \varepsilon)^{1 / 2}$. In other words, beyond Kolmogorov wavenumber, scalar fluctuations are agitated by small scale convection of the velocity field.

Consequently, the scalar spectrum depends on $k, \epsilon_{T}$, and $\tau_{B}$. Dimensional analysis leads to

$$
E_{T}(k, t)=\frac{K_{0}}{3} \varepsilon_{T} \sqrt{\frac{\nu}{\epsilon}} k^{-1} \quad \text { for } \quad k_{\eta}<k<k_{B}
$$

in the so-called viscous-convective zone $k \in\left[k_{\eta} ; k_{B}\right]$. The $k^{-1}$ spectrum was assessed by 

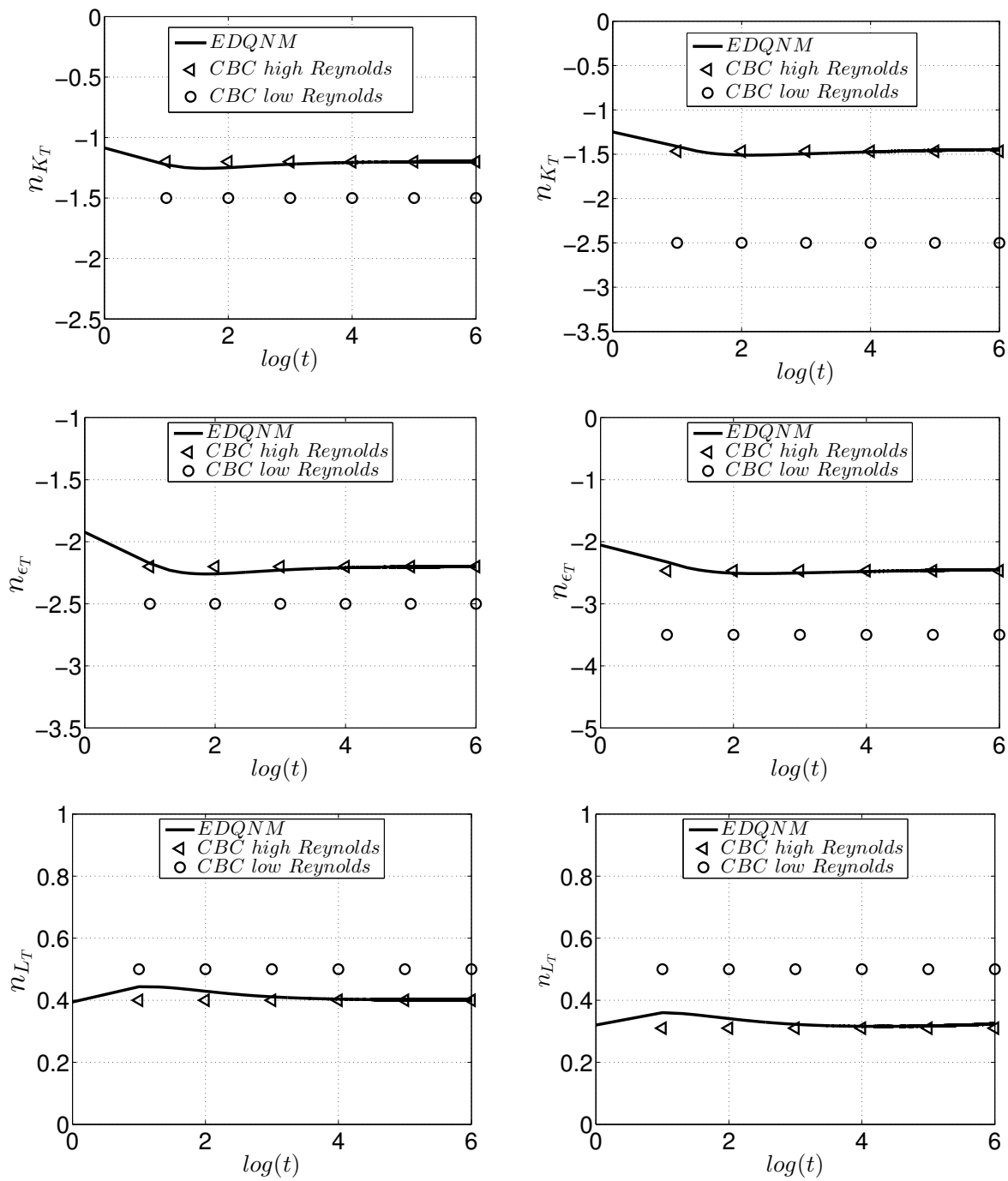

Figure 5. Exponents $n_{K_{T}}, n_{\epsilon_{T}}$ and $n_{L_{T}}$ for $\operatorname{Pr}=10^{-4}$. At left, $s=s^{\prime}=2$. At right $s=s^{\prime}=4$
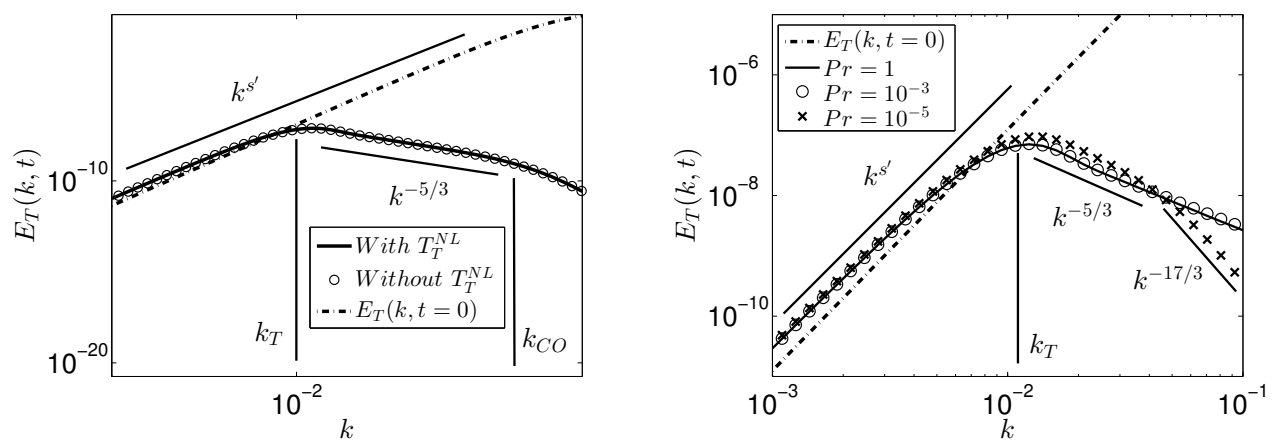

Figure 6. At left, influence of non-local transfers $T_{T}^{N L}$ on large scales of the scalar spectrum $E_{T}$ for $\operatorname{Pr}=10^{-4}$. At right, influence of $\operatorname{Pr}=1,10^{-3}$ and $10^{-5}$ on backscatter. 


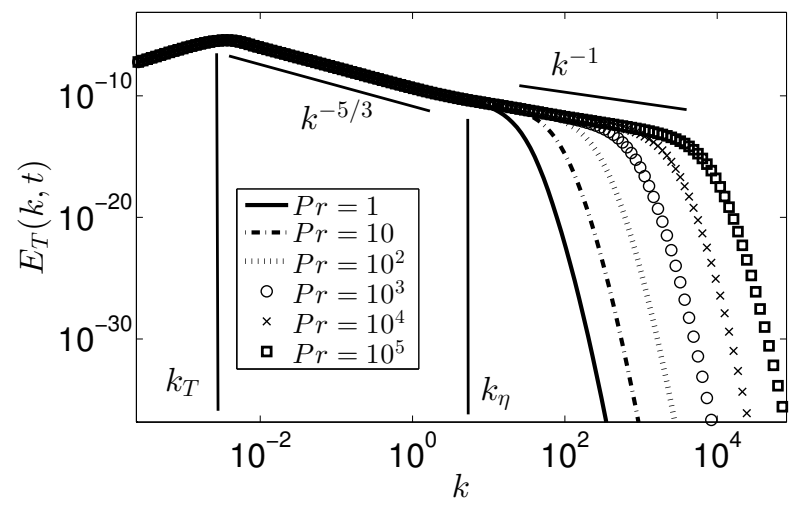

Figure 7. Scalar spectrum $E_{T}$ for $s=s^{\prime}=2$, with $k_{L}$ and $k_{\eta}$ for $\operatorname{Pr}=10^{n}, n \in \llbracket 0 ; 5 \rrbracket$. The Reynolds number is $R e_{\lambda}=500$.

DNS in Yeung et al. (2004). This range is followed by a zone in which an exponential destruction of scalar fluctuations by diffusivity occurs, referred to as the viscous-diffusive range $\left(k \gg k_{B}\right)$.

In this part, the initial Reynolds number is $\operatorname{Re}_{\lambda}(0)=2335$. Unlike the previous case, non-local transfers $T_{T}^{N L}$ defined in $\mathrm{A} 20$ are not negligible. Indeed, as the $k^{-1}$ range is located outside the kinetic spectrum $E$ (beyond $k_{\eta}$ ), local energy transfers are very weak in the viscous-convective range, whereas scalar non-local transfers take energy from large scales and bring it to the very small ones (see Figure 9). Hence, non-local transfers are dominant in the viscous-convective range. Consequently, the scalar spectrum exhibits up to a 2-decades long $k^{-1}$ range for a sufficiently high Prandtl number. The growth of the $k^{-1}$ range when $\operatorname{Pr}$ increases is displayed in Figure 7.

Finally, the decay exponents of $\epsilon_{T}, L_{T}$ and $K_{T}$ are investigated in Figure 8 for $s=$ $s^{\prime}=2$ and $s=s^{\prime}=4$. The scalar exponents clearly follow the extended CBC theory. The result is similar for any $\operatorname{Pr} \geqslant 1$.

\subsubsection{Remarks on large scales and non-local transfers}

As in the previous case $\operatorname{Pr} \ll 1$, EDQNM simulations allow to conclude that for a low diffusivity $\kappa(\operatorname{Pr} \gg 1)$, the Prandlt number does not affect the backscatter for $s=s^{\prime}=4$, neither do the scalar non-local transfers $T_{T}^{N L}$. Their impact on the total transfer (local + non-local) is presented in Figure 9 thanks to the equality

$$
\int_{0}^{\infty} T_{T}^{N L}(k, t) d k=\int_{0}^{\infty} k T_{T}^{N L}(k, t) d(\log k) .
$$

The non-local transfers $T_{T}^{N L}$ take energy from large scales and move it further than local ones, directly in the $k^{-1}$ viscous-convective range.

\section{Transition to low Reynolds and Péclet numbers - Re $\sim P e \sim \mathcal{O}(1)$}

In this section, the transition from high Reynolds to low Reynolds numbers with various Prandtl numbers is investigated. A detailed comparison with experimental results is performed to provide some explanations of the scattering between existing measured scalar decay exponents. 

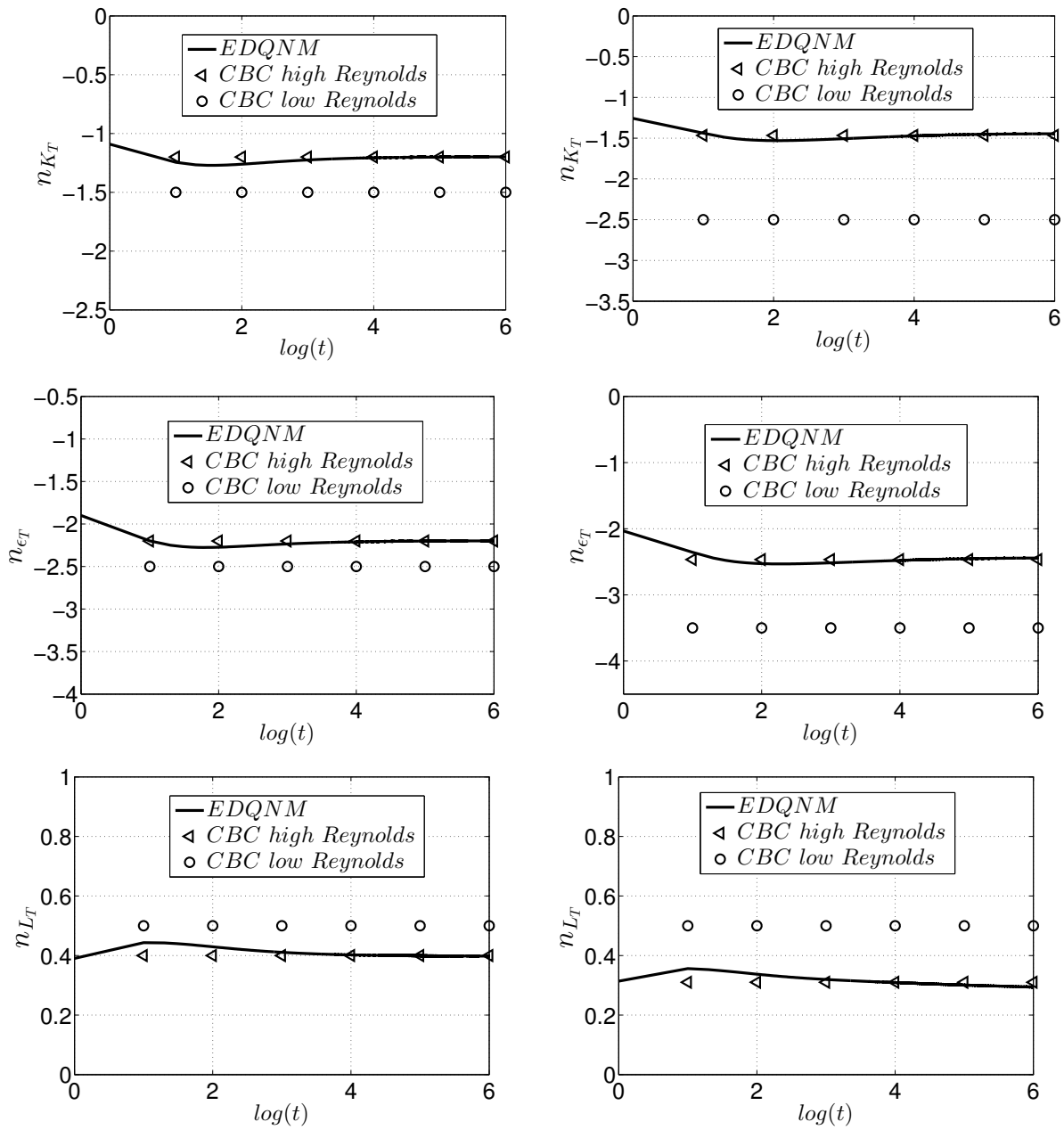

Figure 8. Exponents $n_{K_{T}}, n_{\epsilon_{T}}$ and $n_{L_{T}}$ for $\operatorname{Pr}=10^{4}$. At left, $s=s^{\prime}=2$. At right $s=s^{\prime}=4$

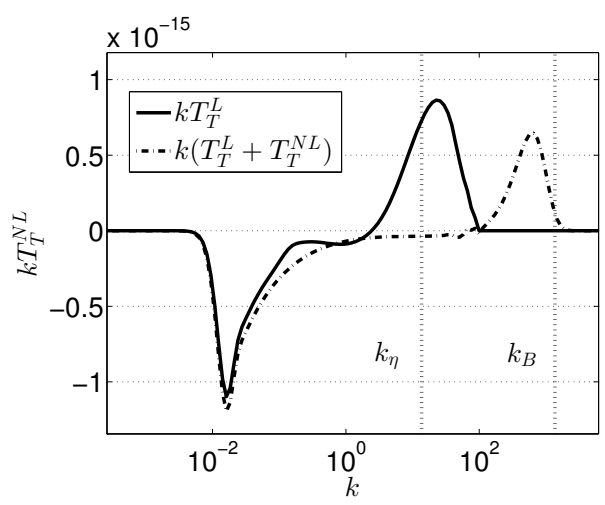

Figure 9. Impact of the scalar non-local transfers $T_{T}^{N L}$ on the total budget term $k\left(T_{T}+T_{T}^{N L}\right)$ for $\operatorname{Pr}=10^{4}$ with $s=s^{\prime}=4$. 


\subsection{Case of reference $\operatorname{Pr}=1$}

\subsubsection{Validation of decay exponents}

The numerical method based on EDQNM analysis allows to illustrate the transition from high to low Reynolds numbers. Several simulations are made until very low Reynolds numbers $R e_{\lambda} \sim 10^{-1}$, starting from $R e_{\lambda}(t=0)=236$. This Reynolds number is high enough to capture the beginning of the transition. As it will be shown, the values of all exponents obtained by EDQNM simulations are all equal (within 1\% error) to their corresponding values in the $\mathrm{CBC}$ analysis. Hence, the scalar extended CBC theory presented in $\$ 3$ gives a satisfactory approximation of decay exponents in the low Reynolds and Péclet regimes.

According to the theory for the case $s=1$, the same exponents for both large and small Reynolds numbers are found. It is only from the shape of the spectrum that these two cases can be distinguished. For infrared exponents $s \geqslant 2$ and $s^{\prime} \geqslant 2$, the Reynolds number decreases over time and inertial ranges of both spectra disappear. This is the low Reynolds numbers regime and the decay exponents $n_{K}$ and $n_{K_{T}}$ converge to the values expected by the extended CBC analysis given in Table 2. This transition grows more rapidly for higher values of infrared spectral slope $s$ and $s^{\prime}$.

Transitions are shown for $s=s^{\prime}=2$ and $s=s^{\prime}=4$ in Figure 10 along with experimental results. The case $s=s^{\prime}=3$, similar to $s=s^{\prime}=2$, is not presented. The small peak that appears around $R e_{\lambda}=5$ is due to the disappearance of the inertial subrange in both $E$ and $E_{T}$. This corresponds to a transition between a regime dominated by inertial effects to a regime dominated by viscous ones. The horizontal lines correspond to theoretical predictions coming from the CBC theory. The experimental scalar decay exponents are displayed for both cases $s=s^{\prime}=2$ and $s=s^{\prime}=4$.

Let's mention that, if one chooses a different initial Reynolds number, higher or lower, the resulting curve would collapse with the present one after two turn-over times $\tau_{0} \simeq$ $K(0) / \epsilon(0)$. Similarly, if different initial spectra $E$ and $E_{T}$ are prescribed, there is only a slight change during the transition. Hence, the present curves for $n_{K}$ and $n_{K_{T}}$ in the transition zone seem quite robust. Nevertheless, there is no clear power law for the decay exponent in the transition.

The critical Reynolds number at which the transition to the low Reynolds regime begins is briefly discussed. Two critical Reynolds numbers are observed for different kinetic and scalar cases, with infrared slope $s=s^{\prime} \in\{2,3,4\}$. The critical Reynolds number for the kinetic case $R e_{\lambda}^{c}$ depends on $s$, and decreases when $s$ increases. Transitions happen at

- Case $s=2: R e_{\lambda}^{c} \approx 38$

- Case $s=3: R e_{\lambda}^{c} \approx 20$

- Case $s=4: R e_{\lambda}^{c} \approx 17$

The transition for the scalar field happens always around $R e_{\lambda_{T}}^{c} \approx 12$. Other scalar exponents such as $n_{\epsilon_{T}}$ and $n_{L_{T}}$ also follow the CBC theory for low Reynolds and Péclet regimes.

As for the low Reynolds numbers regime itself, a validation of the EDQNM closure is proposed in Appendix $\mathrm{B}$ where the mixed-derivative skewness $S_{T}$ is compared with DNS, DIA and TFM (Herring \& Kerr 1982).

\subsubsection{Review of experimental results}

This part aims at providing an overview of experiments for the passive scalar. In such works, the fluid is often air with $P r \simeq 0.7$. In Figure 10, several experimental results regarding the scalar decay exponent $n_{K_{T}}^{\exp }$ are gathered in order to compare them to simulations. These values are mainly provided by Antonia et al. (2013), Lee \& Antonia 

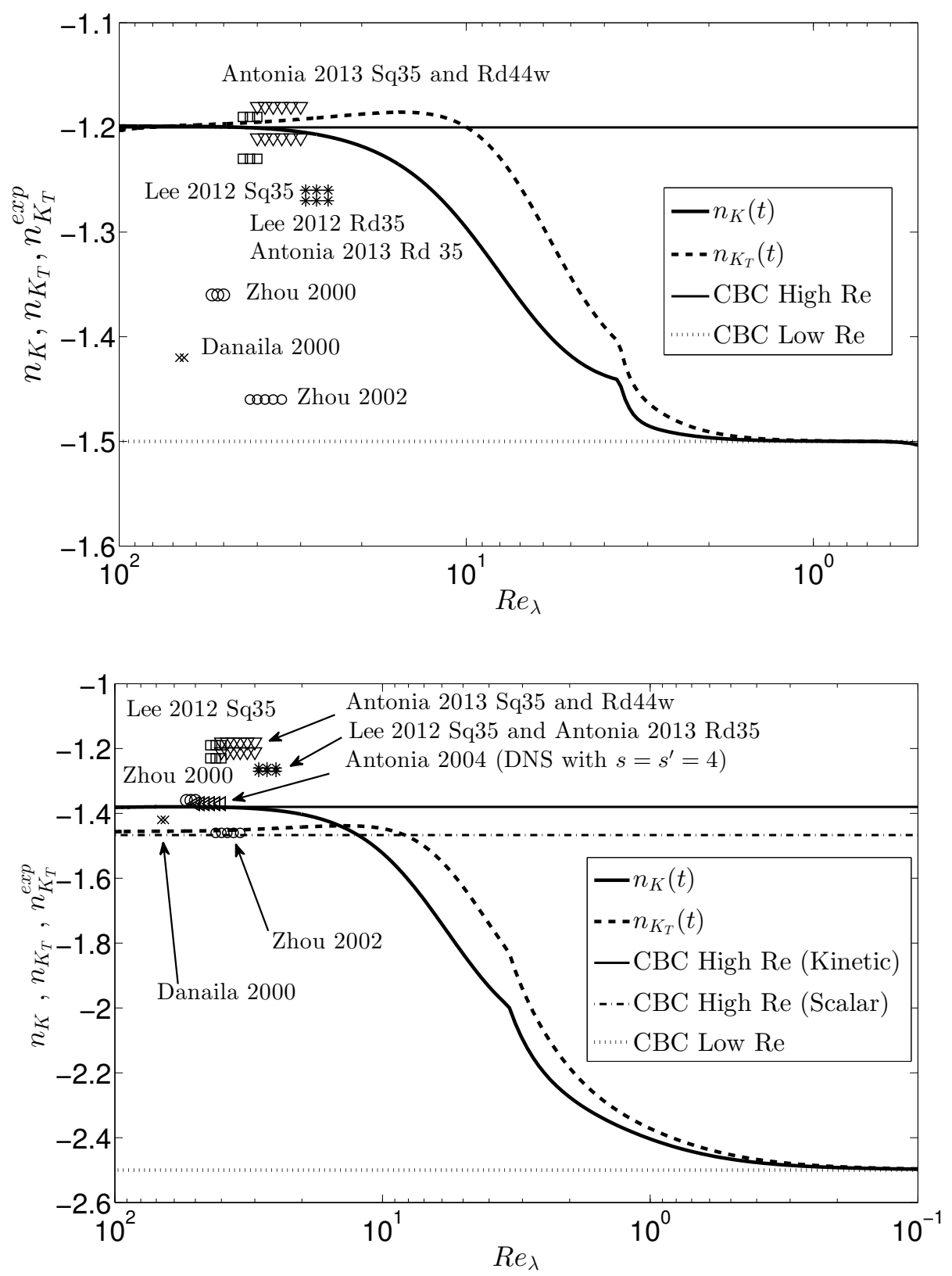

FiguRE 10. Experimental scalar decay exponents $n_{K_{T}}$ at $\operatorname{Pr} \sim 1$ in comparison with high Reynolds CBC theory and EDQNM simulations at $\operatorname{Pr}=1$. Initially, $L(0)=L_{T}(0)=1$. Top, for $s=s^{\prime}=2$. Bottom, for $s=s^{\prime}=4$

(2012), Antonia \& Orlandi (2004) (DNS), Zhou et al. $(2002)$, Zhou et al. $(2000)$ and Danaila et al. (2000). One can note that experimental scalar decay exponents $n_{K_{T}}^{\text {exp }}$ are not close to -1 and that there is no clear tendency toward -1 with increasing Reynolds number. Firstly, it is important here to stress that in these experiments, the Reynolds 
number is rather low $R e_{\lambda} \leqslant 70$. EDQNM simulations show that such Reynolds numbers correspond to the beginning of the transition zone, between high and low Reynolds regimes. This could explain the broad range of scalar decay exponents that have been measured in the past years. The $R e_{\lambda}$ is not high enough to completely match with the high Reynolds and high Péclet regimes, and thus the $n_{K T}^{\exp }$ measured could be misrepresented by the transitional $R e_{\lambda}$-state. Even with DNS, Antonia \& Orlandi (2004) found a $n_{K_{T}}$ which is not the one predicted by the CBC theory. Once again, this might be because of the low $R e_{\lambda}$ and a too low resolution of large scales (which are determinant for the decay exponents) as well. In addition to this, the infrared initial slopes $s$ and $s^{\prime}$ cannot be fixed in grid turbulence. Hence, it is impossible to compare rigorously $n_{K_{T}}^{\exp }$ with the CBC theory.

Nevertheless, interesting results have been obtained experimentally that deserve to be emphasized. First, the scattering of the measured scalar decay exponents $n_{K_{T}}$ is more important than for the kinetic ones $n_{K}$ (Lavoie et al. 2007; Antonia et al. 2013). In addition to the two facts mentioned earlier (low $R e_{\lambda}$ and undefined infrared slopes), $n_{K_{T}}$ varies a lot depending on how the temperature fluctuations are generated. It has been shown (Warhaft \& Lumley 1978, Sreenivasan et al. 1980; Zhou et al. 2000) that the measured value of $n_{K_{T}}$ varies significantly depending on the experimental apparatus: the power used to create the temperature fluctuations or the influence of the measurements origin (the grid, corresponding to the kinetic integral scale $L$, or the heated screen, corresponding to the scalar one $\left.L_{T}\right)$.

Recent experimental works (Zhou et al. (2000, 2002); Lee \& Antonia (2012); Antonia et al. (2013)) have been performed on the scalar decay using similar parameters, which allows to make useful comparisons. More or less all recent experiments were done with a mesh size $M=24.76 \mathrm{~mm}$, an input speed $U \simeq 6 \mathrm{~m} . \mathrm{s}^{-1}$, a mandoline (screen of heated wires) located at $x_{T}=1.5 \mathrm{M}$ and heated with $2 \mathrm{~kW}$ that generates temperature fluctuations $T \in[2 K, 3 K]$. Scalar quantities are measured with the cold wires technique and the Reynolds is such that $R e_{\lambda} \in[30,70]$. However, as soon as $R e_{\lambda}$ remains low, there is an uncertainty due to the transient phase from high Reynolds numbers to low Reynolds numbers. Finally, as the temperature is a passive scalar, it is still submitted to the variations of the kinetic field induced by the kind of grid chosen for experiments (square, round, solidity, active, passive, ....). Therefore, in addition to the moderate $R e_{\lambda}$ effect, the various techniques used to create the turbulent kinetic and scalar fluctuations may be responsible for the scattering.

\subsection{Case $\operatorname{Pr} \neq 1$}

In the previous high Reynolds and high Péclet numbers cases, It has been shown numerically that the Prandtl number does not affect the scalar decay exponents predicted by the CBC theory. The relevant question is now to determine if this behavior is still observed in the case of low Reynolds and low Péclet numbers. An important fact is stressed here: if the Reynolds number is low, it implies a low Péclet regime. In other words, the case of a kinetic field in low Reynolds regime with a scalar field in large Péclet regime does not exist.

\subsubsection{Transition - Case $\operatorname{Pr} \gg 1$}

Firstly, the case $\operatorname{Pr} \gg 1$ is considered: for this kind of fluids, the simulations have to last longer than in the case $\operatorname{Pr} \ll 1$. Indeed, $R e_{\lambda}$ has to decrease a lot in order to reach the low $P e$ regime.

The transition to low Reynolds regime begins when the inertial range $k^{-5 / 3}$ in the kinetic energy spectrum tends to disappear. From this point, the scalar spectrum $E_{T}$ 

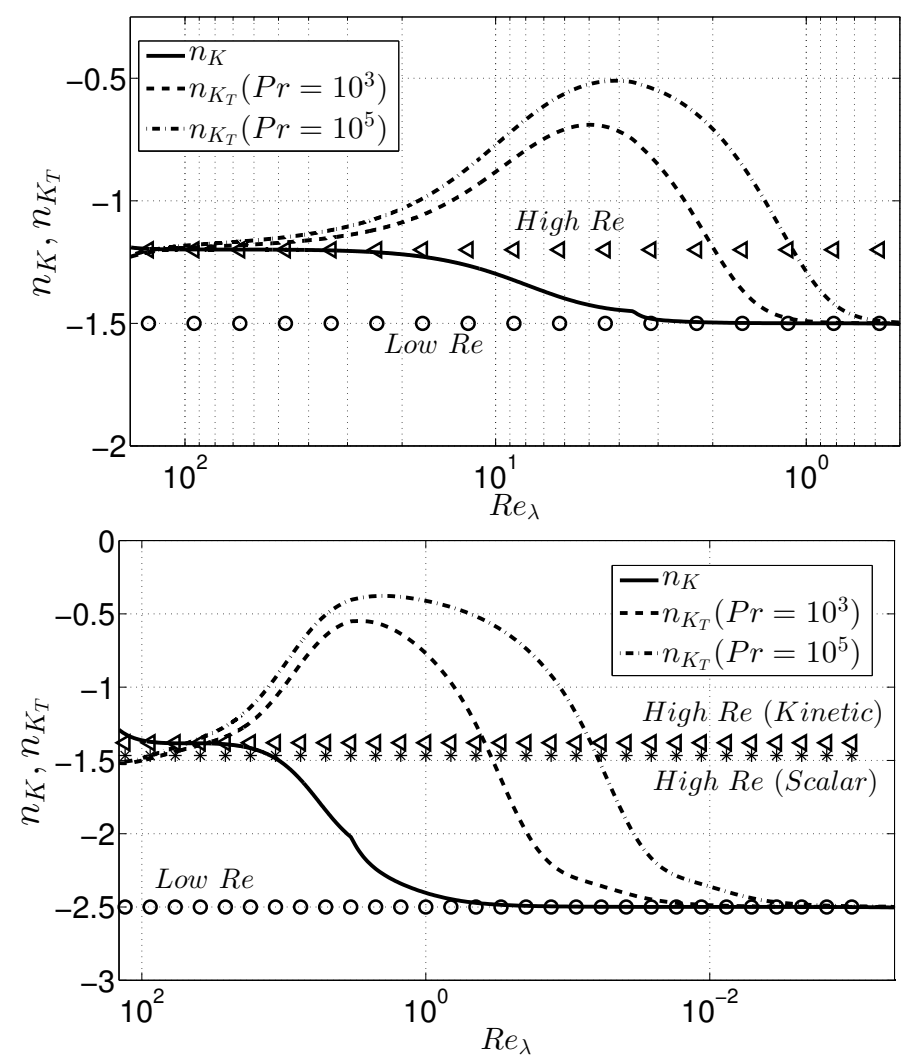

Figure 11. Evolution of kinetic and scalar decay exponents for $\operatorname{Re}_{\lambda}(t=0)=236$ at $\operatorname{Pr}=10^{3}$ and $\operatorname{Pr}=10^{5}$. Top, $s=s^{\prime}=2$. Bottom, $s=s^{\prime}=4$.

still contains a $k^{-1}$ viscous-convective zone wherein the scalar destruction is fairly weak. At the beginning of the transition, with the disappearance of the inertial range, the production of small vortices stops but the friction between small scales creates some temperature fluctuations. Because of this production of temperature variance, the scalar decay slows down and the exponent of scalar variance increases. Then with the disappearance of the viscous-convective zone, the destruction of scalar variance accelerates under the cumulated effects of diffusive and dissipative processes. Hence, the scalar variance decreases with a smaller exponent. Such a behaviour is recovered in Figure 11 where $n_{K_{T}}$ is investigated for various $s=s^{\prime}$ at $\operatorname{Pr}=10^{3}$ and $\operatorname{Pr}=10^{5}$. The first conclusion is that scalar decay exponents provided by the extended CBC theory are still valid in the case of large Prandtl numbers. Only the behaviour of $n_{K_{T}}$, before reaching its asymptotic limit, is modified by $\mathrm{Pr}$. Moreover, two critical Reynolds numbers are observed for the scalar field: a first one from which $n_{K_{T}}$ increases, and a second one from which it decreases, corresponding respectively to the disappearance of the inertial-convective and viscousconvective ranges. The second one is smaller than the one found in the case $\operatorname{Pr}=1$ which was $R e_{\lambda_{T}}^{c} \simeq 12$ in Figure 10. Indeed, as mentioned before, reaching a low Péclet regime with a large Prandtl number is longer than with $\operatorname{Pr}=1$. Hence, $R e_{\lambda}$ has to decrease more. 

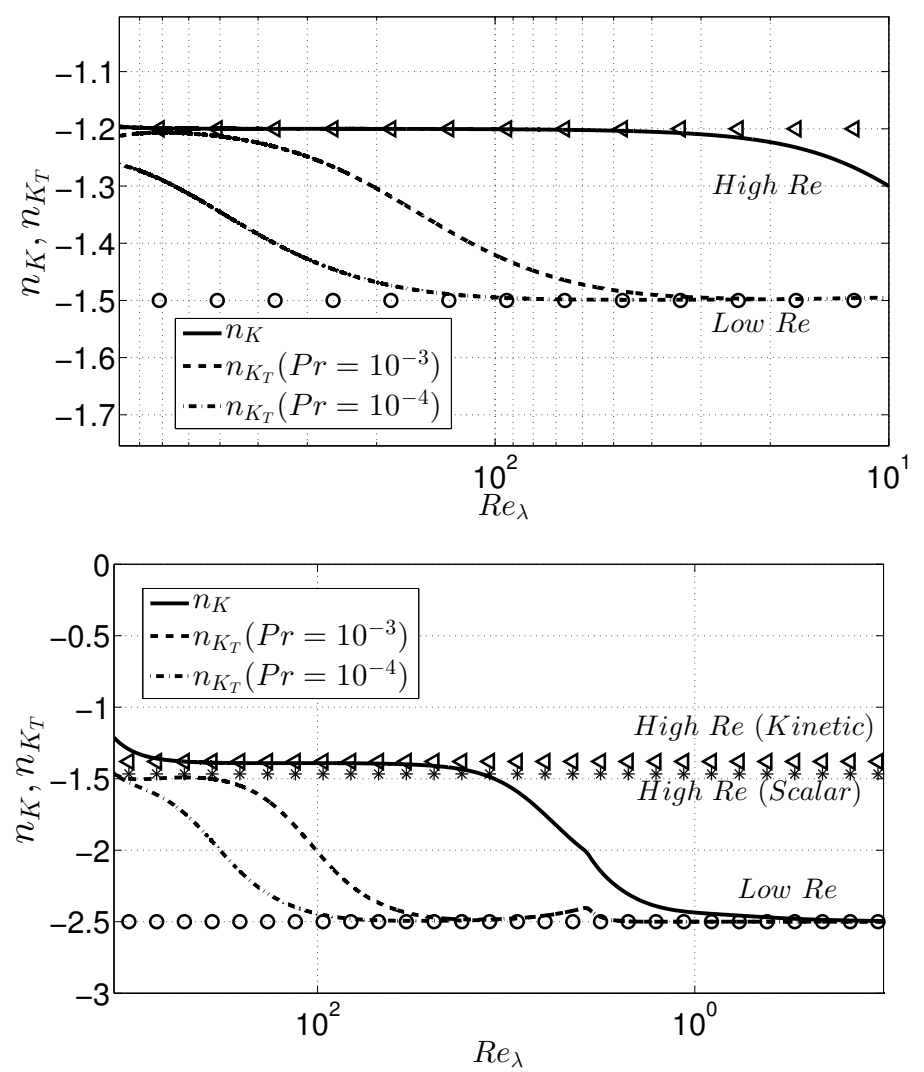

FiguRE 12. Evolution of kinetic and scalar decay exponents for $\operatorname{Re}_{\lambda}(t=0)=1621$ at $\operatorname{Pr}=10^{-3}$ and $\operatorname{Pr}=10^{-4}$. Top, $s=s^{\prime}=2$. Bottom, $s=s^{\prime}=4$.

\subsubsection{Transition - Case $\operatorname{Pr} \ll 1$}

Finally, the case of a strongly diffusive fluid is studied. In Figure 12 scalar decay exponents follow, once again, the extended CBC theory. There is no special behaviour of $n_{K_{T}}$ during the decay: indeed, all scales of the scalar spectrum $E_{T}$ are fully controlled by the kinetic one. In the case $\operatorname{Pr} \ll 1$, the main difference with regard to the case $\operatorname{Pr}=1$ in Figure 10 is that the transition happens earlier: the scalar critical Reynolds number $\operatorname{Re}_{\lambda_{T}}^{c}(\operatorname{Pr} \ll 1)$ is higher than $\operatorname{Re}_{\lambda_{T}}^{c}(\operatorname{Pr}=1)$. As the Prandtl number is very small, the Péclet number $P e=R e P r$ tends faster to the low Péclet regime, and so the critical Reynolds number $R e_{\lambda_{T}}^{c}$ is larger.

It has been shown in $\$ 4$ and $\$ 5$ that whatever the Prandtl number is, the scalar decay exponents follow the extended CBC theory in both high and low Reynolds and Péclet regimes. The Prandtl number does not influence the large scales of the scalar spectrum: therefore, the only relevant parameters that lead the decay of the passive scalar are the infrared slopes $s$ and $s^{\prime}$. 


\section{Study of integral scales $L$ and $L_{T}$}

In this part, the emphasis is put on the evolution of the kinetic and scalar integral scales $L$ and $L_{T}$, defined as

$$
L(t)=\frac{3 \pi}{4 K} \int_{0}^{\infty} k^{-1} E(k, t) d k
$$

and

$$
L_{T}(t)=\frac{\pi}{2 K_{T}} \int_{0}^{\infty} k^{-1} E_{T}(k, t) d k .
$$

\subsection{Evolution of $L_{T}$ - Lesieur's law}

The aim of this part is to show that whatever the Prandtl number is, the relative initial position of the kinetic and scalar integral scales $L(t=0)$ and $L_{T}(t=0)$ does not change the dynamics of the decay. The law provided by Lesieur et al. (1987) gives the temporal evolution of the scalar integral scale

$$
L_{T}(t)=\left(\frac{n_{K}}{n_{K_{T}}}\right)^{3 / 2} L(t)\left(1+B t^{\frac{n_{K}-2}{3}}\right)^{3 / 2},
$$

where $B$ is a constant close to -1 evaluated thanks to initial conditions. The first order of this equation is

$$
\frac{L_{T}}{L}=\left(\frac{n_{K}}{n_{K_{T}}}\right)^{3 / 2}
$$

Such an equation is obtained by dimensional analysis, assuming that in the inertial ranges, $\epsilon$ and $\epsilon_{T}$ scale as

$$
\epsilon=2 K \epsilon^{1 / 3} L^{-2 / 3}, \quad \epsilon_{T}=2 K_{T} \epsilon^{1 / 3} L_{T}^{-2 / 3} .
$$

However, in the low Reynolds and low Péclet regimes, there are no longer inertial and inertial-convective ranges. Therefore $\sqrt{6.3}$ is only valid when $R e \gg 1$ and $P e \gg 1$. In his paper, Lesieur claims that $(6.3)$ is only valid in the case $L_{T}(t=0)<L(t=0)$, meaning that the scalar energy is injected at smaller scales than the kinetic one.

Hereafter, it is shown that all the three different cases $L_{T}(t=0)=L(t=0), L_{T}(t=$ $0)>L(t=0)$ and $L_{T}(t=0)<L(t=0)$ collapse into the same evolution after a transient phase. The cases where $L_{T}(t=0)=10^{2}$ and $10^{-2}$, for $\operatorname{Pr}=10^{-2}$ and $\operatorname{Pr}=10^{2}$ with $L(t=0)=1$ are investigated in Figure 13 . Despite the large final turn-over time $\left(t \sim 10^{11}\right)$, the Reynolds number is still high enough to make sure that there is a clear inertial range $\left(R e_{\lambda} \geqslant 300\right)$. Results before $t=10$ are not shown for the sake of clarity as $L / L_{T}$ is too high. The first conclusion is that the Prandtl number $\operatorname{Pr}$ does not affect the time evolution of $L_{T}$, as predicted by $(6.3)$.

- If $L_{T}(t=0)<L(t=0), L_{T}$ catches up with the case $L_{T}(t=0)=L(t=0)$ after a short transient state.

- If $L_{T}(t=0)>L(t=0), L_{T}$ catches up with the case $L_{T}(t=0)=L(t=0)$ after a long transient state.

- This temporary state occurs whatever the Prandtl number is.

In conclusion, the initial position of $L_{T}$ is not a relevant parameter to study the decay of a passive scalar field because it does not modify its long-time dynamics. This is why, in all the previous computations, both initial temperature and velocity integral scales were assumed to be of the same order. Consequently, the initial peaks of both the kinetic and scalar spectra $k_{L}$ and $k_{T}$ were such that $k_{L}(0)=k_{T}(0)=1$. 

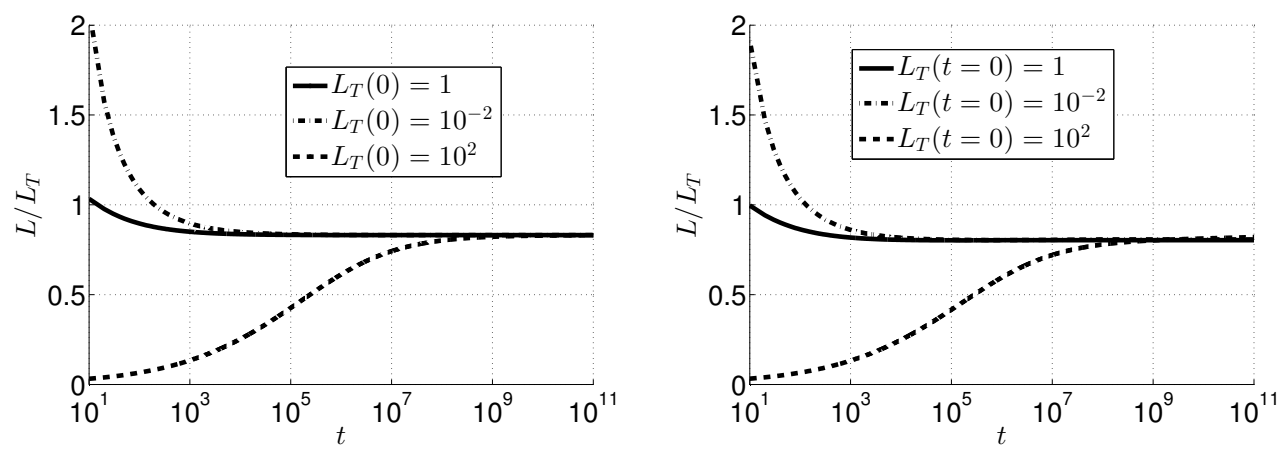

Figure 13. Evolution of $L / L_{T}$ for the three different cases $L_{T}(t=0)=L(t=0)$, $L_{T}(t=0)>L(t=0)$ and $L_{T}(t=0)<L(t=0)$. At left, for $\operatorname{Pr}=10^{-2}$, at right for $\operatorname{Pr}=10^{2}$.

The result presented here is a theoretical one as $L$ and $L_{T}$ collapse for very large turnover times (at least $t \sim 10^{5}$ ). Such large turn-over times are never reached in practice in experiments. Nevertheless, the three cases $L(0)>L_{T}(0), L(0)<L_{T}(0)$ and $L(0)=L_{T}(0)$ are physically meaningful: the first case correspond to the apparatus where the velocity fluctuations are heated after the grid by a mandoline for instance. The second case correspond to a toaster: the laminar field before the grid is heated. The comparison of these two settings has been done by Sirivat \& Warhaft (1983). The latter case correspond to the heated grid (Warhaft \& Lumley 1978).

In the high Reynolds and high Péclet regimes, the common assumptions $k_{L} \simeq 1 / L$ and $k_{T} \simeq 1 / L_{T}$ are made. In the following part, a formula that allows to predict the relative position of $k_{T}$ and $k_{L}$ is provided, valid only in the high Reynolds and Péclet regimes.

\subsection{Prediction of $k_{L}$ and $k_{T}$}

Here, a law able to predict the relative position of the peaks of both kinetic and scalar spectra, respectively $k_{L}$ and $k_{T}$, is derived. This law is valid in high Reynolds and Péclet regimes, as soon as kinetic and scalar integrated quantities decay according to the CBC theory. We define the ratio

$$
\beta=\frac{k_{T}}{k_{L}} .
$$

The assumption $\beta=1$ is commonly made, but this value is not obtained with EDQNM simulations. Moreover, the fact that $k_{T} \neq k_{L}$ has already been observed in experiments: Warhaft \& Lumley (1978) and Sreenivasan et al. (1980) noted that there was a link between the scalar decay exponent $n_{K_{T}}$ and the ratio $k_{T} / k_{L}=\beta$. Zhou et al. (2000) also made the observation and proposed a correlation. Nevertheless, as the infrared exponents are unknown in experiments, it is impossible to make relevant comparisons. This is the reason why an analytical law linking $\beta$ and $n_{K_{T}}$ in the high Reynolds and Péclet regimes is proposed here. For convenience purposes in the calculations, piecewise spectra are used

$$
E(k, t)=\left\{\begin{array}{l}
A(t) k^{s} \quad \text { for } \quad k<k_{L} \\
K_{0} k^{-5 / 3} \epsilon^{2 / 3} \quad \text { for } k \in\left[k_{L} ; k_{\eta}\right]
\end{array}\right.
$$

and

$$
E_{T}(k, t)=\left\{\begin{array}{l}
A_{T}(t) k^{s^{\prime}} \quad \text { for } k<k_{T} \\
K_{C O} \epsilon_{T} \epsilon^{-1 / 3} k^{-5 / 3} \text { for } k_{T}<k<k_{\eta} \\
K_{B} \epsilon_{T} \sqrt{\frac{\nu}{\epsilon}} k^{-1} \text { for } k_{\eta}<k<k_{B}
\end{array}\right.
$$




$\begin{array}{cccc}\text { Cases } s, s^{\prime} & \beta_{t h} & \beta_{N} & \text { Relative error } \\ s=s^{\prime}=1 & 2 / 3 & 0.6684 & 0.26 \% \\ s=s^{\prime}=2 & 2 / 3 & 0.6684 & 0.26 \% \\ s=s^{\prime}=3 & 2 / 3 & 0.6684 & 0.26 \% \\ s=s^{\prime}=4 & 0.7165 & 0.7645 & 6.2 \% \\ s=1, s^{\prime}=4 & 1.418 & 1.496 & 5.21 \% \\ s=2, s^{\prime}=3 & 0.9104 & 0.8744 & 3.95 \% \\ s=2, s^{\prime}=4 & 1.07 & 1 & 6.64 \% \\ s=3, s^{\prime}=2 & 0.4882 & 0.5110 & 4.46 \% \\ s=4, s^{\prime}=1 & 0.31 & 0.299 & 3.5 \% \\ s=4, s^{\prime}=2 & 0.430 & 0.4468 & 3.76 \%\end{array}$

TABLE 5 . Values of the ratio $\beta=k_{T} / k_{L}$ and relative errors given for several simulations with $s=s^{\prime}$ and $s \neq s^{\prime}, \forall \operatorname{Pr} \in\left[10^{-5} ; 10^{5}\right]$

for $\operatorname{Pr} \geqslant 1$, and

$$
E_{T}(k, t)=\left\{\begin{array}{l}
A_{T}(t) k^{s^{\prime}} \text { for } k<k_{T} \\
K_{C O} \epsilon_{T} \epsilon^{-1 / 3} k^{-5 / 3} \text { for } k_{T}<k<k_{C O} \\
K_{B} \epsilon_{T} a^{-3} \epsilon^{2 / 3} k^{-17 / 3} \text { for } k_{C O}<k<k_{\eta}
\end{array}\right.
$$

for $\operatorname{Pr} \leqslant 1$. The computation of the ratio $L / L_{T}$ yields

$$
\frac{L}{L_{T}}=\frac{3 C^{\prime} C_{T}}{2 C C_{T}^{\prime}} \beta
$$

where $C=\frac{1}{1+s-p}+\frac{3}{2}, C_{T}=\frac{1}{1+s^{\prime}-p^{\prime}}+\frac{3}{2}, C^{\prime}=\frac{1}{s-p}+\frac{3}{5}$ and $C^{\prime}=\frac{1}{s^{\prime}-p}+\frac{3}{5}$. Then, using Lesieur model $\frac{L}{L_{T}}=\left(\frac{n_{K_{T}}}{n_{K}}\right)^{3 / 2}$, an explicit law for $\beta$ is obtained

$$
\beta=\frac{2}{3}\left(\frac{s-p}{s^{\prime}-p^{\prime}}\right)\left(\frac{1+s^{\prime}-p^{\prime}}{1+s-p}\right)\left(\frac{n_{K_{T}}}{n_{K}}\right)^{3 / 2} .
$$

The law 6.8 is valid as soon as there are inertial and inertial-convective subranges in $E$ and $E_{T}$, i.e. in high Reynolds and Péclet numbers. In order to show the relevance of this formula, $\beta$ is computed in several cases with $s=s^{\prime}$ and with $s \neq s^{\prime}$. All results are gathered in Table 5 where $\beta_{N}$ refers to the numerical results and $\beta_{t h}$ to the theoretical ones coming from (6.8). This formula provides less than $1 \%$ error when $s=s^{\prime} \leqslant 3$ and a maximum of $6.64 \%$ when $s^{\prime}=4$. Finally, the law 6.8 does not depend on the Prandtl number: all cases in Table 5 give the same value for $\operatorname{Pr}=10^{3}$ and $\operatorname{Pr}=10^{-3}$, consistent with the fact that $\operatorname{Pr}$ does not affect large scales.

This law completes the work of Ristorcelli (2006) regarding the time scale ratio $r$ : indeed, in the analytical computation of $r$, the ratio $k_{T} / k_{L}$ is introduced but no explicit formula is given.

\section{Conclusion}

This paper aims at characterizing the decay of a passive scalar field in homogeneous isotropic turbulence. To this end, results coming from EDQNM simulations are compared to the Comte-Bellot and Corrsin (CBC) analysis, which is a simplified approach based on dimensional analysis. So far, the CBC analysis was giving the exact values of the decay exponents obtained by EDQNM simulations for the infrared slopes $s \in\{1,2,3,4\}$ 
in the kinetic case. Based on the results of EDQNM simulations, an extension of the CBC analysis for the passive scalar decay is presented. This corrects all scalar decay exponents of integrated quantities when the scalar infrared spectral slope $s^{\prime}=4$.

The main result of this paper is that the theoretical scalar decay exponents of the extended CBC theory are valid whatever the Prandtl number is (very large, very small, and close to one) in both high and low Reynolds and Péclet regimes. A broad range of Prandtl numbers spanning from $\operatorname{Pr}=10^{-5}$ to $\operatorname{Pr}=10^{5}$ was used in simulations to obtain such a general result. The main consequence of this study is that the Prandtl number only affects small scales of the scalar spectrum $E_{T}$. The large scales $\left(k<k_{L}\right)$ depend only on the infrared slopes $s$ and $s^{\prime}$ which is confirmed by the match of theoretical CBC exponents and EDQNM simulations.

The impact of the relative initial position of the scalar integral scale $L_{T}(t=0)$ with respect to the kinetic one $L(t=0)$ in the case where $\operatorname{Re} \gg 1$ and $\operatorname{Pe}=\operatorname{Re} \operatorname{Pr} \gg 1$ is also studied. Neither the Prandtl number nor the initial position of $L_{T}$ affect the final result of the passive scalar decay as soon as the high Reynolds and high Péclet regimes are maintained. This result is important for a complete understanding of the passive scalar decay, and shows that $L_{T}(t=0)$ is not a necessary parameter in long time simulations.

In other words, the problem of the passive scalar decaying turbulence has been simplified, reducing the relevant parameters from $\left(R e, P r, s, s^{\prime}, L_{T}(t=0)\right)$ to $\left(P e, s, s^{\prime}\right)$. In the continuity of this study, a law able to predict the relative position of the peaks of both kinetic and scalar spectra $k_{L}$ and $k_{T}$ was proposed. This law derived for the high Reynolds and high Péclet regimes is valid whatever the Prandtl number and the infrared slopes $s$ and $s^{\prime}$ are.

In conclusion, this paper can be seen as a general study of the passive scalar decay in the framework of the homogeneous isotropic freely decaying turbulence. The consistency of the results over a wide range of Prandtl numbers shows that the decay of the passive scalar is driven only by the most energetic large scales of the initial spectra. Neither the initial position of the scalar integral scale nor the Prandtl number are observed to affect the final result of the decay.

\section{Appendix A. EDQNM Modelling for the kinetic and scalar fields}

The spectral counterpart of the Navier-Stokes equation is written as $N S(k)$

$$
\frac{\partial u(k)}{\partial t}=\widehat{u u}-\nu k^{2} u(k)
$$

on the plane perpendicular to $k$ in order to erase the pressure term. $u$ is the fluctuating velocity field, $k$ the wavenumber, and $\widehat{()}$ the convolution product. If this equation is summed in two points $u(p) N S(k)$ and $u(k) N S(p)$ and averaged $<()>$, the spectral equation of the correlation in two points is obtained

$$
\left[\frac{\partial}{\partial t}+\nu\left(k^{2}+p^{2}\right)\right]\langle u(k) u(p)\rangle=\langle u u u\rangle .
$$

With $k=p$, the so-called Lin equation is recovered

$$
\frac{\partial E(k, t)}{\partial t}+2 \nu k^{2} E(k, t)=T(k, t)
$$

where $T(k, t)$ denotes the energy transfer between different turbulent scales. This term can be split into two types of transfers, local transfers $T^{L}$ representing energy exchanges between two vortices of similar size, and non-local transfers $T^{N L}$ representing exchanges 
between eddies of different size. Indeed, the kinetic energy can be transferred from one scale to another. The energy transfer of turbulent fluctuations created at a given scale to smaller ones is called the direct cascade of kinetic energy. The inverse cascade refers to the transfer occurring from small to large scales (Lesieur \& Schertzer (1978)).

If the process from A 1 to A 2 is repeated for three points correlation, the following equation is obtained

$$
\left[\frac{\partial}{\partial t}+\nu\left(k^{2}+p^{2}+q^{2}\right)\right]\langle u(k) u(p) u(q)\rangle=\langle u u u u\rangle .
$$

One can always redo the transition from equation (A 2) to (A 4), but after each iteration, a higher order term appears. This is the closure problem.

In the quasi-normal approximation, the fourth-order moment of the equation $(\mathrm{A} 4 \mathrm{C})$ is assumed to behave as a Gaussian random variable and can be written as the sum of the product of second order moments

$$
\left[\frac{d}{d t}+\nu\left(k^{2}+p^{2}+q^{2}\right)\right]\langle u(k) u(p) u(q)\rangle=\sum\langle u u\rangle\langle u u\rangle .
$$

Orszag (1970) proposed a compensation of the error due to this approximation by introducing a characteristic eddy-damping rate $\mu_{k p q}$ of the third order moment associated to the triad $[k, p, q]$

$$
\left[\frac{\partial}{\partial t}+\nu\left(k^{2}+p^{2}+q^{2}\right)+\mu_{k p q}\right]\langle u(k) u(p) u(q)\rangle=\sum\langle u u\rangle\langle u u\rangle
$$

with

$$
\mu_{k p q}=\nu\left(k^{2}+p^{2}+q^{2}\right)+\eta^{E D}(k, t)+\eta^{E D}(p, t)+\eta^{E D}(q, t)
$$

and

$$
\eta^{E D}(k, t)=a_{1} \sqrt{\int_{0}^{k} p^{2} E(p, t) d p}
$$

where $a_{1}=0.36$ so that the Kolmogorov constant $K_{0}=1.40$ is recovered. With this triad notation, the kinetic energy spectrum evolution equation reads

$$
\frac{\partial E(k, t)}{\partial t}+2 \nu k^{2} E(k, t)=\int_{0}^{t} d \tau \int_{p+q=k}\left[\exp \left(-\nu\left(k^{2}+p^{2}+q^{2}\right)(t-\tau)\right)\right] \sum\langle u u\rangle\langle u u\rangle d p
$$

The Markovianisation consists in assuming that the exponential term in the integral does not depend on time so that integrals in A 7) can be permuted. In addition, the characteristic time of the exponential is assumed to vary less than the one linked to $\sum\langle u u\rangle\langle u u\rangle$. Therefore, the formula (A 7) can be simplified and written as

$$
\begin{gathered}
\frac{\partial E(k, t)}{\partial t}+2 \nu k^{2} E(k, t)=\int_{p+q=k} \theta_{k p q} \sum\langle u u\rangle\langle u u\rangle(t) d p \\
\theta_{k p q}=\int_{0}^{t}\left[\exp \left(-\nu\left(k^{2}+p^{2}+q^{2}\right)(t-\tau)\right)\right] d \tau=\theta_{k p q}=\frac{1-e^{-\mu_{k p q} t}}{\mu_{k p q}}
\end{gathered}
$$

Finally the Lin equation reads

$$
\frac{\partial E(k, t)}{\partial t}+2 \nu k^{2} E(k, t)=T^{N L}(k, t)+T^{L}(k, t)
$$


with the kinetic local transfers

$$
T^{L}(k, t)=\int_{\Delta_{k}} \theta_{k p q}\left(x y+z^{3}\right) \frac{E(q, t)}{q}\left[k^{2} E(p, t)-p^{2} E(k, t)\right] d p d q
$$

and the non-local ones

$$
T^{N L}(k, t)=-\frac{\partial \Pi^{N L}}{\partial k}=-\frac{\partial\left(\Pi^{N L^{+}}-\Pi^{N L^{-}}\right)}{\partial k},
$$

where $\Pi^{N L}$ is the non-local flux

$$
\begin{aligned}
\Pi^{N L}(k, t) & =\left[\frac{2}{15} k\left(E(k)-k \frac{\partial E}{\partial k}\right) \int_{0}^{a k} \theta_{k k q} q^{2} E(q) d q+\frac{2}{15} \frac{E(k)^{2}}{k} \int_{0}^{a k} \theta_{k k q} q^{4} d q\right] \\
& -\left[-\frac{2}{15} \int_{0}^{k} k^{\prime 2} E\left(k^{\prime}\right) d k^{\prime} \int_{\sup \left(k, k^{\prime} / a\right)}^{\infty} \theta_{k^{\prime} p p}\left(5 E(p)+p \frac{\partial E}{\partial p}\right) d p\right. \\
+ & \left.\frac{14}{15} \int_{0}^{k} k^{\prime 4} d k^{\prime} \int_{\sup \left(k, k^{\prime} / a\right)}^{\infty} \theta_{k^{\prime} p p} \frac{E(p)^{2}}{p^{2}} d p\right] .
\end{aligned}
$$

Due to the logarithmic discretization, the elongated triads such that

$$
\frac{\inf (k, p, q)}{\sup (k, p, q)} \leqslant a
$$

are not taken into account in the numerically computed energy transfers (except for isosceles interactions $k=p=q$ ). To get rid of this difficulty a method has been developed by Lesieur \& Schertzer (1978) and also applied to two dimensional turbulence by Pouquet et al. (1975). In practice the non-loical parameter $a$ is taken of order $\mathcal{O}\left(10^{-1}\right)$ to evaluate correctly the non-local transfers due to the triadic interactions. In our simulations, the non-local parameter is $a=10^{1 / 17}-1=0.1450$, where 17 is the number of points per decade.

The process is similar for the passive scalar. Starting from the spectral equation

$$
\frac{\partial T(k)}{\partial t}+\kappa k^{2} T(k)=\widehat{T u}
$$

the characteristic third-order correlation time is

$$
\begin{array}{r}
\theta_{k p q}^{T}=\frac{1-\exp \left[-\left(\kappa\left(k^{2}+p^{2}\right)+\nu q^{2}+\mu^{\prime}(k)+\mu^{\prime}(p)+\mu^{\prime \prime}(q)\right) t\right]}{\kappa\left(k^{2}+p^{2}\right)+\nu q^{2}+\mu^{\prime}(k)+\mu^{\prime}(p)+\mu^{\prime \prime}(q)} \\
\mu^{\prime}(k)=a_{2} \sqrt{\int_{0}^{k} p^{2} E(p, t) d p}, \quad \mu^{\prime \prime}(k)=a_{3} \sqrt{\int_{0}^{k} p^{2} E(p, t) d p}
\end{array}
$$

with $a_{2}=0$ and $a_{3}=1.3$ (see the discussion in Lesieur (2008); Herring et al. $(1982)$ ). The choice of $a_{2}$ and $a_{3}$ is based on experimental considerations so that the Corrsin constant $K_{C O}=0.66$ is recovered. This choice is also convenient for numerical computation. Nevertheless, a different setting (for instance $a_{1}=a_{2}=a_{3}$ would lead to the same result). Finally, the so-called scalar Lin equation is obtained

$$
\frac{\partial E_{T}(k, t)}{\partial t}+2 \kappa k^{2} E_{T}(k, t)=T_{T}^{L}(k, t)+T_{T}^{N L}(k, t)
$$


with the scalar local transfers

$$
T_{T}^{L}(k)=\int_{\Delta_{k}} \theta_{k p q}^{T} \frac{k}{p q}\left(1-y^{2}\right) E(q)\left[k^{2} E_{T}(p)-p^{2} E_{T}(k)\right] d p d q
$$

and the non-local ones

$$
T_{T}^{N L}(k, t)=-\frac{\partial \Pi_{T}^{N L}}{\partial k}=-\frac{\partial\left(\Pi_{T}^{N L^{+}}-\Pi_{T}^{N L^{-}}\right)}{\partial k},
$$

where $\Pi_{T}^{N L}$ is the non-local scalar flux verifying

$$
\begin{aligned}
\Pi_{T}^{N L}(k, t) & =\left[k\left(2 E_{T}(k)-k \frac{\partial E_{T}}{\partial k}\right) \frac{2}{15} \int_{0}^{a k} \theta_{k k q}^{T} q^{2} E(q) d q\right. \\
& \left.+\frac{1}{4} E(k) \int_{0}^{a k} \theta_{k k q}^{T} q^{3} E_{T}(q) d q-\frac{1}{4} \frac{E(k) E_{T}(k)}{k^{2}} \int_{0}^{a k} \theta_{k k q}^{T} q^{5} d q\right] \\
& -\left[-\frac{4}{3} \int_{0}^{k} k^{\prime 2} E_{T}\left(k^{\prime}\right) d k^{\prime} \int_{\sup \left(k, k^{\prime} / a\right)}^{\infty} \theta_{k^{\prime} p p}^{T} E(p) d p\right. \\
& \left.+\frac{4}{3} \int_{0}^{k} k^{\prime 4} d k^{\prime} \int_{\sup \left(k, k^{\prime} / a\right)}^{\infty} \theta_{k^{\prime} p p}^{T} \frac{E(p) E_{T}(p)}{p^{2}} d p\right] .
\end{aligned}
$$

The first r.h.s term of $\Pi_{T}^{N L}$ is responsible for the $k^{-1}$ viscous-convective subrange.

\section{Appendix B. Mixed scalar skewness $S_{T}$ in low Reynolds regime}

EDQNM has already been used to study third-order statistics in homogeneous isotropic turbulence for the velocity field, for instance by Meldi \& Sagaut (2013) where the velocity skewness and palinstrophy were investigated. Here a brief validation of EDQNM closure is presented for the mixed scalar skewness

$$
S_{T}=\frac{<(\partial u / \partial x)(\partial \theta / \partial x)^{2}>}{<\sqrt{(\partial u / \partial x)^{2}}><(\partial \theta / \partial x)^{2}>}
$$

for low Reynolds numbers. A comparison with DNS of two-point closures has been performed in this framework by Herring \& Kerr (1982), where the TFM and DIA are investigated. Here, it is shown that EDQNM captures well the behavior of third-order statistics.

The scalar Lin equation reads

$$
\left(\frac{\partial}{\partial t}+2 \kappa k^{2}\right) E_{T}(k, t)=T_{T}(k, t)
$$

where $T_{T}$ is the total non-linear scalar transfer. One can deduce the evolution equation of the scalar dissipation rate $\epsilon_{T}$, as done by Ristorcelli (2006)

$$
\frac{\partial \epsilon_{T}}{\partial t}=-\left(\sqrt{\frac{5}{3}} S_{T} \sqrt{R e_{T}}+\frac{5}{9} r G_{T}\right) \frac{\epsilon \epsilon_{T}}{K} .
$$




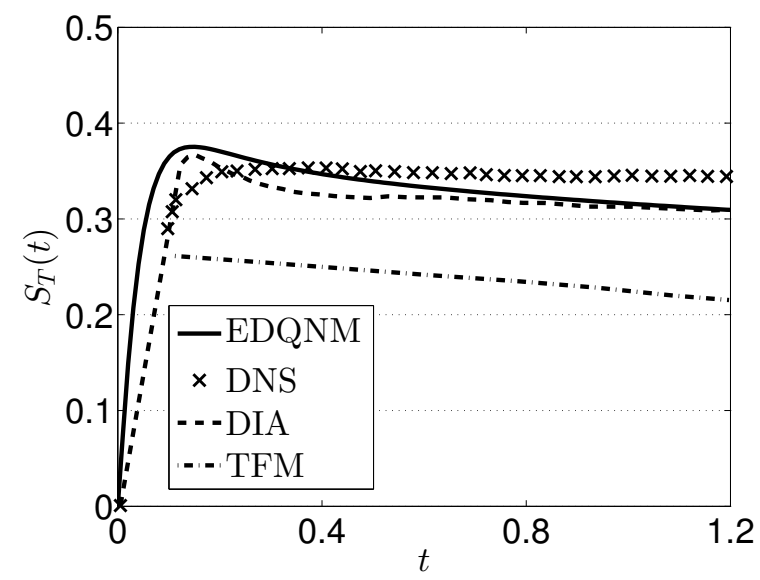

Figure 14. Comparison of the mixed scalar skewness computed with EDQNM with DNS, DIA and TFM of Herring \& Kerr (1982).

$S_{T}$ is the mixed scalar skewness

$$
S_{T}(t)=-\sqrt{\frac{3}{10}} \frac{\int_{0}^{\infty} k^{2} T_{T} d k}{\sqrt{\int_{0}^{\infty} k^{2} E d k} \int_{0}^{\infty} k^{2} E_{T} d k},
$$

$G_{T}$ is the scalar palinstrophy

$$
G_{T}(t)=\frac{18}{5} \kappa \frac{K_{T}}{\epsilon_{T}} \frac{\int_{0}^{\infty} k^{4} E_{T} d k}{\int_{0}^{\infty} k^{2} E_{T} d k},
$$

$R e_{T}$ is the turbulent Reynolds number $R e_{T}=K^{2} /(\nu \epsilon)$, and $r$ is the time scales ratio $\left(K \epsilon_{T}\right) /\left(K_{T} \epsilon\right)$.

Figure 2 of Herring and Kerr shows that $R e_{\lambda}$ decreases from 12 to 6 with initial Batchelor $k^{4}$ spectrum. In the present Figure $14 . S_{T}$ computed with EDQNM is displayed. Same initial conditions as in Herring and Kerr have been used. The final value obtained with EDQNM is in good agreement with DIA, close to DNS, and does not present the same issue as TFM which slightly under-estimates $S_{T}$ for low Reynolds numbers. The initial growth phase is well-captured with regard to DNS as well.

\section{REFERENCES}

Antonia, R. A., Lee, S. K., Duenidi, L. \& Danaila, L. 2013 Invariants for slightly heated decaying grid turbulence. Journal of Fluid Mechanics 727, 379-406.

Antonia, R. A. \& Orlandi, P. 2004 Similarity of decaying isotropic turbulence with a passive scalar. Journal of Fluid Mechanics 505, 123-151.

Batchelor, G. K., Howells, I. D. \& Townsend, A. A. 1958 Small-scale variation of convected quantities like temperature in turbulent fluid. Journal of Fluid Mechanics 5.

Batchelor, G. K. \& Proudman, I. 1956 The large-scale structure of homogeneous turbulence. Phil. Trans. R. Soc. Lond. A 248, 369-405.

Briard, A. \& GOMEZ, T. 2015 A passive scalar convective-diffusive subrange for low prandtl numbers in isotropic turbulence. Physical Review E 91.

Chasnov, J., Canuto, V. M. \& Rogallo, R. S. 1988 Turbulence spectrum of a passive temperature field: results of a numerical simulation. Physics of Fluids 31, 2065-2067.

Comte-Bellot, G. \& Corrsin, S. 1966 The use of a contraction to improve the isotropy of a grid generated turbulence. J. Fluid Mech 25, 657-682. 
Corrsin, S. 1951 The decay of isotropic temperature fluctuations in an isotropic turbulence. Journal of Aerosol Science 18, 417.

Danaila, L., Zhou, T., Anselmet, F. \& Antonia, R. A. 2000 Calibration of a temperature dissipation probe in decaying grid turbulence. Experiments in Fluids 28, 45-50.

Eyink, G. L. \& Thomson, D. J. 2000 Free decay of turbulence and breakdown of self-similarity. Physics of Fluids 12, 477-479.

Goto, S. \& KIDA, S. 1999 Passive scalarspectrum in isentropic turbulence: Prediction by the lagrangian direct-interaction approximation. Physics of Fluids $\mathbf{1 1 .}$

Granatstein, V. L. \& Buchsbaum, S. J. 1966 Fluctuation spectrum of a plasma additive in a turbulence gas. Physical Review Letters 16, 504-507.

Grant, H. L., Hughes, B. A., Vogel, W. M. \& Moilliet, A. 1968 The spectrum of temperature fluctuations in turbulent flow. Journal of Fluid Mechanics 34, 423-442.

Herring, J. R. \& KerR, R. M. 1982 Comparison of direct numerical simulations with predictions of two-point closures for isotropic turbulence convecting a passive scalar. Journal of Fluid Mechanics 118, 205-219.

Herring, J. R., Schertzer, D., Lesieur, M., Newman, G. R., Chollet, J. P. \& LARCHEVEQUE, M. 1982 A comparative assessment of spectral closures as applied to passive scalar diffusion. Journal of Fluid Mechanics 124, 411-437.

KANEDA, Y. 1986 Inertial range structure of turbulent velocity and scalar fields in a lagrangian renormalized approximation. Physics of Fluids 29.

Kraichnan, R. H. 1968 Small-scale structure of a scalar field convected by turbulence. Physics of Fluids 11 (5), 945-953.

Lavoie, P., Duenidi, L. \& Antonia, R. A. 2007 Effects of initial conditions in decaying turbulence generated by passive grids. Journal of Fluid Mechanics 585, 395-420.

Lee, S. K. \& Antonia, R. A. 2012 Scaling range of velocity and passive scalar spectra in grid turbulence. Physics of Fluids 24.

Leith, C.E. 1971 Atmospheric predictability and two-dimensional turbulence. J. Atmos. Sci. 28, 145.

Lesieur, M. 2008 Turbulence in fluids 4th Edn.. Dordrecht: Martinus nijhoff publishers.

Lesieur, M., Montmory, C. \& Chollet, J.P. 1987 The decay of kinetic energy and temperature variance in threedimensional isotropic turbulence. Physics of Fluids $\mathbf{3 0}$.

Lesieur, M. \& OssiA, S. 2000 3D isotropic turbulence at very high reynolds numbers: Edqnm study. J. Fluid Mech 1 (17).

Lesieur, M. \& Rogallo, R. 1989 Large-eddy simulation of passive-scalar diffusion in isotropic turbulence. Physics of Fluids A 1,718-722.

Lesieur, M. \& Schertzer, D. 1978 Amortissement auto similaire d'une turbulence à grand nombre de reynolds. Journal de Mecanique $\mathbf{1 7}$.

LiN, S. C. \& LiN, S. C. 1973 Turbulence spectrum of a passive temperature field: results of a numerical simulation. Physics of Fluids 16, 1587-1598.

Llor, A. \& Soulard, O. 2013 Comment on energy spectra at low wavenumbers in homogeneous incompressible turbulence [phys. lett. a 375 (2011) 2850]. Physics Letters A 377, 1157-1159.

Lumley, J. L. 1970 Stochastic Tools in Turbulence. Academic Press.

Marinis, D., Chibbaro, S., Meldi, M. \& Sagaut, P. 2013 Temperature dynamics in decaying isotropic turbulence with joule heat production. Journal of Fluid Mechanics $\mathbf{7 2 4}$.

Meldi, M. \& Sagaut, P. 2012 On non-self-similar regimes in homogeneous isotropic turbulence decay. J. Fluid Mech 711, 364-393.

Meldi, M. \& Sagaut, P. 2013 Further insights into self-similarity and self-preservation in freely decaying isotropic turbulence. Journal of Turbulence 14, 24-53.

Meyers, J. \& Meneveau, C. 2008 A functional form of the energy spectrum parametrizing bottleneck and intermittency effects. Physics of Fluids 20.

Mons, V., Chassaing, J. C., Gomez, T. \& Sagaut, P. 2014 Is isotropic turbulence decay governed by asymptotic behavior of large scales? an eddy-damped quasi-normal markovianbased data assimilation study. Physics of Fluids 26.

Nelkin, M. \& KerR, R. M. 1981 Decay of scalar variance in terms of a modified richardson law of pair dispersion. Physics of Fluids 24, 1754.

OrszaG, S.A. 1970 Analytical theories of turbulence. J. Fluid Mech p. 363. 
Orszag, S.A. 1977 The statistical theory of turbulence. In Fluid Dynamics (ed. A. Balian \& J.L. Peube), pp. 237-374. Gordon and Breach, New York.

Pope, S. B. 2000 Turbulent Flows. Cambridge University Press.

Pouquet, A., Lesieur, M., Andre, J. C. \& Basdevant, C. 1975 Evolution of high reynolds number two-dimensional turbulence. Journal of Fluid Mechanics $\mathbf{7 7}$.

Ristorcelli, J. R. 2006 Passive scalar mixing: Analytic study of time scale ratio, variance, and mix rate. Physics of Fluids 18.

Ristorcelli, J. R. \& Livescu, D. 2004 Decay of isotropic turbulence: Fixed points and solutions for $g \sim R_{\lambda}$ palinstrophy. Physics of Fluids 16, 3487 .

Rust, J. H. \& Sesonske, A. 1966 Turbulent temperature fluctuations in mercury and ethylene glycol in pipe flow. International of Heat Mass Tranfer 9, 215-227.

SAfFman, P. G. 1967 The large-scale structure of homogeneous turbulence. Journal of Fluid Mechanics 27, 551-593.

Sagaut, P. \& Cambon, C. 2008 Homogeneous Turbulence Dynamics. Palaiseau,France: Cambridge University Press.

Sirivat, A. \& WARHAFT, Z. 1983 The effect of a passive cross-stream temperature gradient on the evolution of temperature variance and heat flux in grid turbulence. Journal of Fluid Mechanics 128, 323-346.

SReEnivasAn, K. R. 1996 The passive scalar spectrum and the obukhov-corrsin constant. Physics of Fluids 8 (1), 189-196.

Sreenivasan, K. R., Tavoularis, S., Henry, R. \& Corrsin, S. 1980 Temperature fluctuations and scales in grid generated turbulence. Journal of Fluid Mechanics 100, 597-621.

Tennekes, H. \& Lumley, J.L. 1972 A first course in Turbulence. Cambridge: MIT Press.

WARhaft, Z. \& LumLEY, J. L. 1978 An experimental study of the decay of temperature fluctuations in grid generated turbulence. Journal of Fluid Mechanics 88, 659-684.

Yeung, P. K., Xu, S., Donzis, D. A. \& Sreenivasan, K. R. 2004 Simulations of threedimensional turbulent mixing for schmidt numbers of the order 1000. Flow, Turbulence and Combustion 72, 333-347.

Zhou, T., Antonia, R. A. \& ChuA, L. P. 2002 Performance of a probe for measuring turbulent energy and temperature dissipation rates. Experiments in Fluids 33, 334-345.

Zhou, T., Antonia, R. A., Danaila, L. \& Anselmet, F. 2000 Transport equations for the mean energy and temperature dissipation rates grid turbulence. Experiments in Fluids $\mathbf{2 8}$, $143-151$. 